Mitteilungen der Österreichischen Geographischen Gesellschaft,

155. Jg. (Jahresband), Wien 2013, S. 303-304

\title{
Berichte und Kleine Mitteilungen
}

\section{IGU Regional Conference 2013}

\section{Kyōto, 4. bis 9. August 2013}

Peter JORDAN, Wien*

Die Internationale Geographische Union (International Geographical Union, IGU) veranstaltet alle vier Jahre einen Weltkongress. Der letzte hat im Vorjahr (2012) in Köln stattgefunden (vgl. Nissel \& Embleton-Hamann 2012), der nächste wird im Jahr 2016 in Peking [Beijing] ausgerichtet werden. Zwischendurch finden aber nun schon jährlich sogenannte „Regionalkonferenzen" statt, die trotz ihrer örtlich einschränkenden Bezeichnung inhaltlich kaum weniger global sind als die Weltkongresse und auch der Teilnehmerzahl nach beinahe deren Größe erreichen. Was sie vielleicht am meisten von den Weltkongressen unterscheidet, ist der Umstand, dass Teilnehmer und Vorträge aus bzw. über die Weltregion des jeweiligen Austragungsortes besonders zahlreich sind.

So war das auch bei der Regionalkonferenz der IGU in Kyōto: Von den nach offiziellen Angaben 1.434 Teilnehmern kam sicher der Großteil aus Ostasien; und japanische oder ostasiatische sowie pazifische Themen standen bei den Plenarsitzungen wie auch in vielen Panels im Vordergrund. Das Generalthema der Konferenz „Traditional Wisdom and Modern Knowledge for the Earth's Future" nahm auf die traditionsreichen Kulturen Ostasiens Bezug, setzte der ganzen Themenvielfalt der Geographie aber auch keinerlei Grenzen.

Die japanische Note der Konferenz, die im Kyōto International Conference Centre, dem Ort der UN Framework Convention of Climate Change im Jahr 1997 und der Unterzeichnung der berühmten Kyōto-Protokolle stattfand (vgl. Abb. 1), kam natürlich bei der Eröffnung besonders

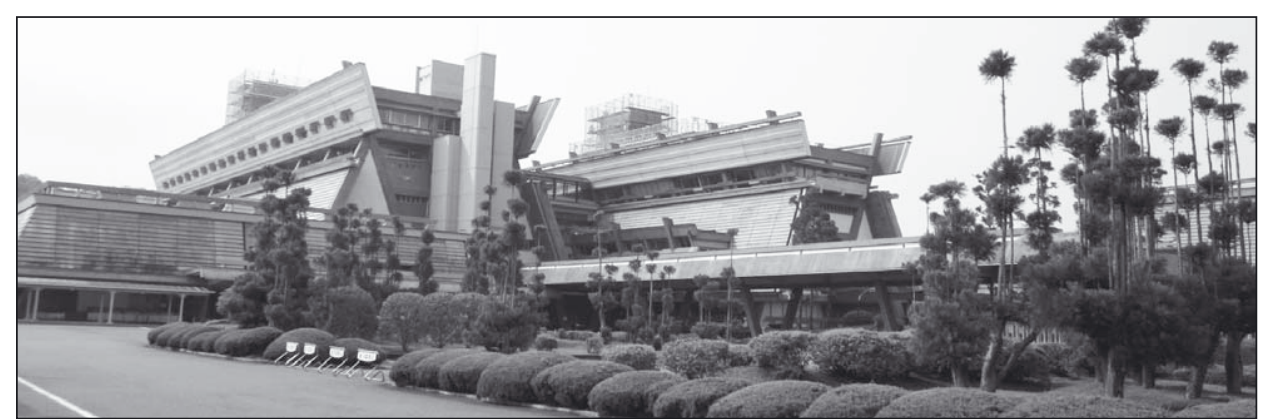

Abb.1: Kyōto International Conference Centre (Foto: Peter JoRDAN)

* HR Prof.h.c. Univ.-Doz. Dr. Peter JoRDAN, Institut für Stadt- und Regionalforschung, Österreichische Akademie der Wissenschaften, Postgasse 7/4/2,A-1010 Wien; E-Mail: peter.jordan@ oeaw.ac.at, http://www.oeaw.ac.at/isr 
zur Geltung: Diese wurde durch die Anwesenheit des japanischen Prinzenpaares, Prinz Akishino und Prinzessin Kıко, ausgezeichnet. Prinz Aкısнino hielt dabei auch eine Rede, in der er auf seine wissenschaftlichen Arbeiten hinwies und deren Nähe zur Geographie betonte. Die Teilnahme des Prinzenpaares gab auch Anlass zu besonderen Sicherheitsmaßnahmen: Man musste schon lang vor Beginn der Eröffnungsveranstaltung im Saal sein und wurde eigens kontrolliert. Die Eröffnungssitzung startete auf die Sekunde pünktlich und lief „wie am Schnürchen“ ab. Pünktlichkeit, straffe Organisation und zu 100\% planmäßiger Ablauf waren aber nicht nur Charakteristika der Eröffnungssitzung, sondern der ganzen Konferenz. Sie war auch die erste unter der Präsidentschaft des neuen IGU-Präsidenten Vladimir KoLosov vom Institut für Geographie der Russischen Akademie der Wissenschaften in Moskau [Moskva], der in Köln in dieser Funktion Ron F. AbLER (USA) gefolgt war.

Die neun Plenarsitzungen waren überwiegend Umweltthemen gewidmet. Besonderes Interesse fand die Sitzung zur Nuklearkatastrophe des Jahres 2011 von Fukushima mit dem vorausgegangenen Erdbeben und dem Tsunami. Man erfuhr viel über das schon gut ausgebaut gewesene, eigentlich beispielhafte Erdbeben- und Tsunami-Warnsystem, über präventive Evakuierungsmaßnahmen, über die dadurch wohl verhinderten noch größeren Schäden, aber auch über den genauen Ablauf der Katastrophe, die dennoch eingetretenen gewaltigen Schäden und über die voraussichtlichen Folgewirkungen auf Gesellschaft und Wirtschaft. Es wurde deutlich,

dass es selbst einem bestens organisierten, hochtechnisierten und wirtschaftlich starken Land wie Japan nicht gelingt, derartiger Naturgewalten und einer Nuklearkatastrophe Herr zu werden.

Es ist einem einzelnen Teilnehmer kaum möglich, die thematische Vielfalt der zahlreichen parallelen Panels zu überblicken und wissenschaftlich einzuordnen. Es sei daher nur die recht starke Präsenz der östlichen Nachbarländer Österreichs (Tschechiens, der Slowakei, Ungarns und Sloweniens) sowie der Schweiz und Italiens hervorgehoben. So waren aus Tschechien bzw. der Slowakei auch Dušan Drbohlav (Prag [Praha]) und Vladimír Ira (Pressburg [Bratislava]), die beiden Mitglieder des Herausgeberkomitees der MÖGG, mit Vorträgen vertreten; aus der Schweiz war es unter anderen die Österreicherin Doris WastL-W ALter (Bern).

Die Teilnahme aus Österreich bewegte sich in engen Grenzen. Andreas KocH vom Fachbereich Geographie und Geologie der Universität Salzburg hielt einen Vortrag zum Thema „ICT and health care effort in sparsely populated areas. An example from a northern Swedish town“ und der Verfasser leitete eine Sitzung der Joint ICA/IGU Working Group/Commission on Toponyms und präsentierte ein Paper zur Frage „Is , exonym' an appropriate term for features beyond any sovereignty?"“

Das Rahmenprogramm bot außer einer Ice Breaker Party, dem Festbankett und einer japanischen Tee-Zeremonie zahlreiche Exkursionen in die Stadt Kyōto und in dessen nähere und weitere Umgebung. Diese litten allerdings unter den auch für japanische Verhältnisse außergewöhnlichen Augusttemperaturen von an die $40^{\circ} \mathrm{C}$ schon am Morgen und noch am Abend bei hoher Luftfeuchtigkeit, die den Aufenthalt außerhalb klimatisierter Räume - zumindest für Mitteleuropäer - äußerst mühsam machten. Der Reiz Kyōtos, das unter den ansonsten sehr modernen und gleichförmigen größeren japanischen Städten sicher zu den baulich und kunstgeschichtlich interessantesten zählt, wäre sonst wohl stärker zur Geltung gekommen.

\section{Literaturhinweis:}

Nissel H., Embleton-Hamann Chr. (2012), Down to Earth - IGC Cologne 2012. 32. Weltkongress der Geographie in Köln. August 2012. In: Mitteilungen der Österreichischen Geographischen Gesellschaft, 154, S. 341-346. 
Mitteilungen der Österreichischen Geographischen Gesellschaft,

155. Jg. (Jahresband), Wien 2013, S. 305-316

\title{
„Johann Oskar ${ }^{61)}$ Friedrich Simony zum 200. Geburtstag
}

\author{
Wolfgang Rudolf KaINRATH, Wien*
}

mit 6 Abb. im Text

\section{INHALT}

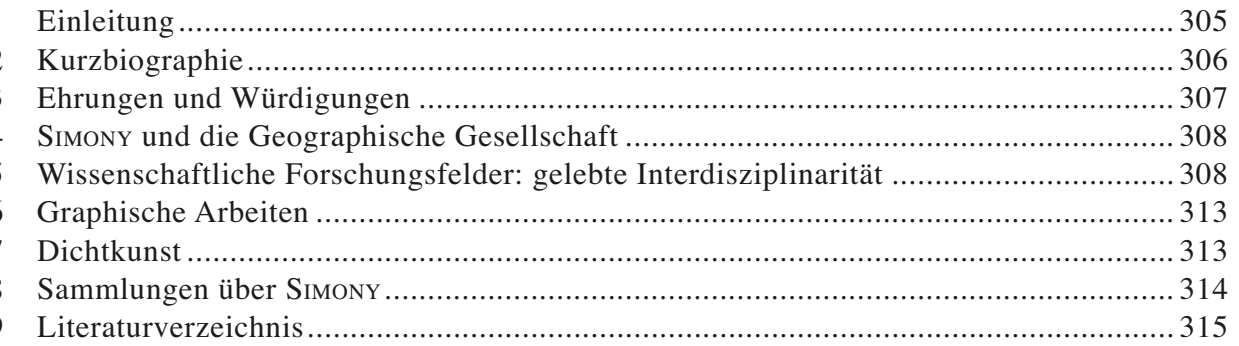

\section{Einleitung}

Siebzehn Jahre nach dem Gedenken zum 100. Todestag an den Dachsteinforscher, ersten Ordinarius für Geographie in Wien und multidisziplinären Forscher gibt sich die Österreichische Geographische Gesellschaft die Ehre, das Lebenswerk Friedrich SimONYs aus heutiger Sicht zu würdigen. Auch die Frage nach der Nachhaltigkeit seiner Arbeiten darf gestellt werden: Ist alles, was vor 200 Jahren geschaffen wurde, heute nur noch Erinnerung und Romantik?

Viel hat sich in den letzten zwei Jahrzehnten getan, was es rechtfertigt, Leben und Werk erneut zu betrachten. Insbesondere die Möglichkeiten, ohne großen Aufwand den Weg der Digitalisierung zur Langzeitarchivierung zu nützen und bislang nicht oder nur in bescheidener technischer Qualität gedruckte Werke der Öffentlichkeit zugänglich zu machen, erfordern ständig neue Recherchen.

Es sind im Laufe der Jahre auch noch interessante Details zum Leben und Wirken SimonYs aufgetaucht, die in der folgenden Kurzbiographie berücksichtigt werden. Detaillierte Darstellungen von Leben und Werk liegen mit dem „Friedrich-Simony-Gedenkband“ (FISCHER, NAGL \& Wohlschlägl 1996) und dem Ausstellungskatalog „Ein Leben für den Dachstein“ (SPETA 1996) seit 1996 vor.

1) Eintrag in der Familienchronik der Familie Krackowizer: ,Johann Oskar Friedrich Simony kk. UniversitätsProfessor in Wien“. Handschrift „Die Familie Krackowizer“, als „Concept“ ausgeführt, Privatbesitz im Familienarchiv von Ferdinand Krackowizer (1851-1929) und OÖ Landesarchiv Sign. S662.

* Wolfgang Rudolf KAINRATH, Universitätsbibliothek, Universität Wien, Universitätsring 1, A-1010 Wien; E-Mail: wolfgang.kainrath@univie.ac.at, http://www.univie.ac.at/wolfgang.kainrath 


\section{Kurzbiographie}

Simony (Szimonj) Friedrich wurde am 30. November 1813 in Hrochowteinitz [Hrochův Týnec] bei Chrudim in Ostböhmen [Východočechy] als unehelicher Sohn bis heute nicht zweifelsfrei bekannter Eltern geboren. Der Vater war - so wird vermutet - ungarischer Armeearzt. Spekulationen über seine Mutter sind noch unsicherer, sie verstirbt jedenfalls früh. Seine Kindheit und Jugend verlief keineswegs glücklich und geborgen, er wurde von seinen Großeltern und anderen Verwandten aufgezogen.

Er besuchte von 1825 bis 1828 ein Ordensgymnasium in Nikolsburg [Mikulov] in Mähren [Morava], absolvierte danach die Apothekergehilfenlehre im damals zu Ungarn gehörenden, heute in der Slowakei gelegenen Trentschin [Trenčín], arbeitete dann als Laborant in Znaim [Znojmo] und kam im Jahr 1835 nach Wien, wo er das Magisterrigorosum aus Pharmazie (kein akademischer Titel im heutigen Sinn) ablegte. Danach studierte er Naturwissenschaften.

Bereits 1840 begann er mit der geomorphologischen und glaziologischen Erforschung des Dachsteins, die in seinem dreibändigen Lebenswerk „Das Dachsteingebiet“ (1889-95) vollendet wurde. Heute unvorstellbar sind die Bedingungen, unter welchen er drei Dezembertage (1842) und zwei Septembernächte (1843) auf dem Dachstein verbringt. Zuvor hatte er mit Helfern aus Hallstatt mit finanzieller Unterstützung von Erzherzog Johann einen Klettersteig gebaut und im Wildkar eine einfache, steinerne Schutzhütte errichtet (heute noch als „Hotel Simony“ zu sehen).

$\mathrm{Ab} 1844$ führte er als einer der Wegbereiter der Limnologie Lotungen in vielen Alpenseen durch, deren Ergebnisse im „Atlas der österreichischen Alpenseen“ (2 Bände, vgl. PEncK \& RichTER 1895-96) dokumentiert sind.

Im Jahr 1848 wurde er Kustos im Naturhistorischen Landesmuseum in Klagenfurt, 1849 Chefgeologe der Sektion V der Geologischen Reichsanstalt und schließlich 1851 ordentlicher Professor für die gesamte Geographie an der Universität Wien, womit die Errichtung der Lehrkanzel und die Begründung des heutigen Institutes für Geographie und Regionalforschung einhergingen.

SimONY war auch Mitbegründer der k.k. Geographischen Gesellschaft in Wien (4.11.1856) und des Österreichischen Alpenvereins, der am 19.11.1862 in der Akademie der Wissenschaften in Wien durch Paul Grohmann, Edmund Mojsisovics von Mojsvár und Guido von Sommaruga (damals Studenten), unterstützt von Eduard Suess, Friedrich Simony, Johann Josef PeYritsch und Anton von RuthNER, gegründet wurde.

Am 2.7.1851 heiratete Simony Amalia Krackowizer (*2.7.1821 in Wels, †14.5.1877 in Wien) in Thalheim bei Wels. Sein Privatleben war im weiteren Verlauf von Schicksalsschlägen geprägt. Eine Tochter starb bald nach der Geburt, sein Sohn Arthur $(* 1854)$, der ebenso wie sein Sohn Oskar kinderlos blieb, starb 23-jährig - es ist nicht klar, ob als junger Arzt (vgl. LeHr 1996, S. 16) oder ohne abgeschlossenes Medizinstudium und ,süchtig“ (vgl. SPETA 1996, S. 7) - kurz nach Amalia‘s Tod.

Zwei private Freundschaften sind bemerkenswert und bedeutsam: Adalbert STIFTER, der Simony als Vorlage für Heinrich Drendorf in seinem Roman „Nachsommer“ nimmt - aus deren privater Korrespondenz erfahren wir einige der insgesamt spärlichen privaten Details - und Johann Georg Ramsauer (1795-1874), den er in Hallstatt als Bergmann kennengelernt hat und der ihn als Entdecker des Hallstätter Gräberfeldes mit dem Fund vertraut macht. SimonYs Bergführer in Hallstatt, Johann W ALLNER (1802-1878), gehört ebenfalls zu den sehr wichtigen Personen in seinem Leben. In Hallstatt, das ab 1842 zu einem zweiten Lebensmittelpunkt wird, pflegt er auch einen guten Teil der sozialen Kontakte. Ausführlich nachzulesen ist über diesen Lebensabschnitt bei LEHR (1996).

Seine letzten Lebensjahre verbringt Simony an der Seite einer Frau, die ihn pflegt und die bisher kaum Erwähnung fand: Johanna Heigenhauser (1855-1942) aus Sankt Gallen (in der 

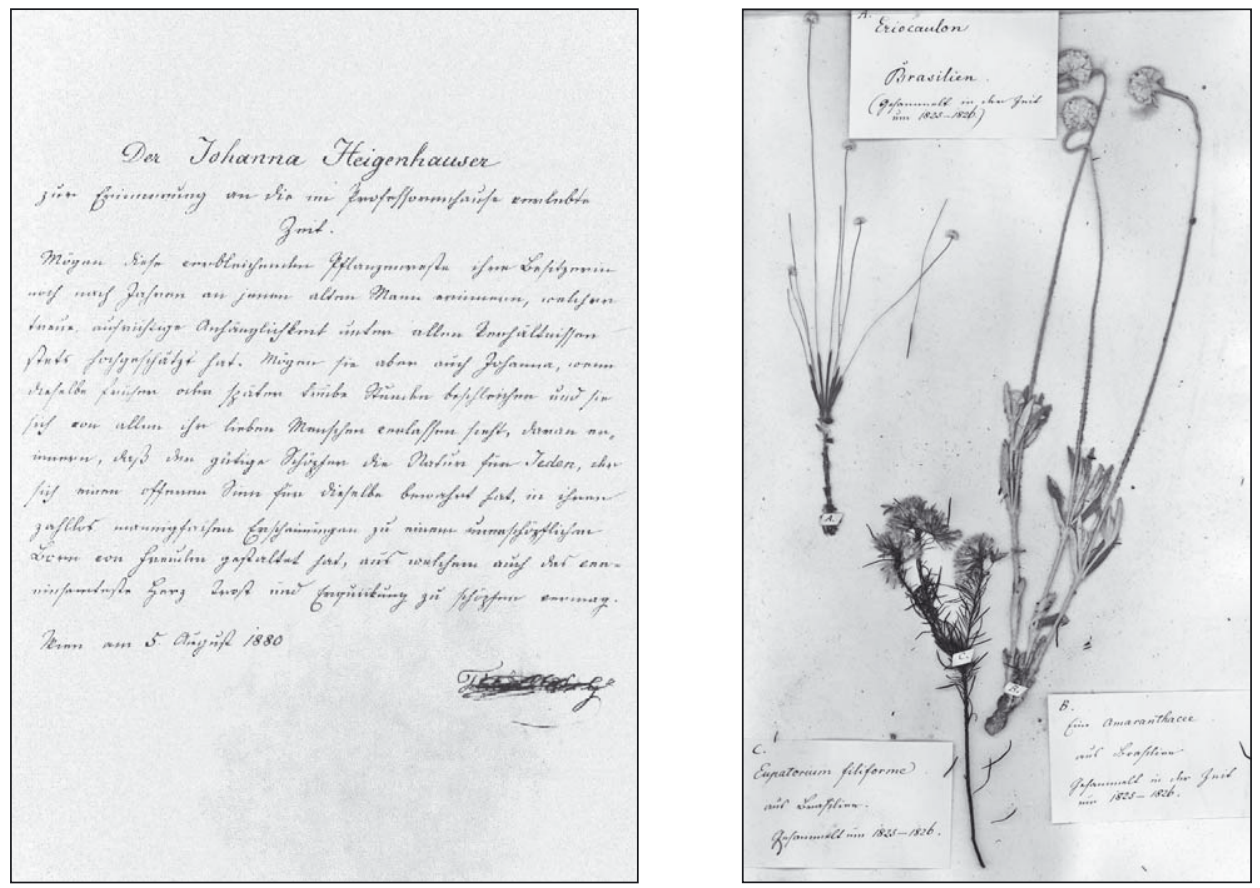

Quelle: Sammlung „Teilnachlass F. Simony“, Universität Wien

Abb. 1: Blatt A des Herbariums mit der Widmung „Der Johanna Heigenhauser zur Erinnerung an die im Professorenhause erlebte Zeit"

Steiermark). Hinweise auf sie finden sich unter den Bezeichnungen "Simonyfräulein" oder „Pflegerin von Professor Simony“ im Historischen Alpenarchiv. ${ }^{2)}$ Ihr widmet er 1880 ein Herbarium (vgl. Abb. 1). Simony stirbt am 20.7.1896 in Sankt Gallen im steirischen Ennsgebiet.

\section{Ehrungen und Würdigungen}

Bereits zu Lebzeiten erhielt SimONY die Ehrenbürgerschaft von Hallstatt (14.5.1876), 1885 wurde er zum k.k. Hofrat ernannt. Im Jahr 1869 benannte der Mineraloge Gustav TscherMAK (1836-1927) ein vermeintlich neu entdecktes Gestein als Simonyit. Da es sich dabei um den bereits bekannten Blödit (wasserhaltiges Natrium-Magnesium-Sulfat) handelte, wird der Name nur selten als Synonym verwendet.

Im Jahr 1883 wurde Simony Ehrenmitglied der k.k. Geographischen Gesellschaft, von der er 1896 mit die Franz-von-Hauer-Medaille ausgezeichnet wurde. Im Jahr 1907 wurde in WienWähring die Simonygasse nach ihm benannt, 2013 dort eine Gedenktafel angebracht. Im Jahr 1970 erfolgte die Enthüllung einer Gedenktafel am Wohn- und Sterbehaus Friedrich SimonYs in Sankt Gallen.

Zum 100. Todestag im Jahr 1996 erschienen zahlreiche Publikationen, etwa der „FriedrichSimony-Gedenkband“ mit den Vorträgen des Gedenkkolloquiums am 26.6.1996 am Institut für

2) Historisches Alpenarchiv, Sektion Austria, Sign. OeAV SE/12/311. 
Geographie in Wien. Außerdem gab es vom 23.9. bis 18.10.1996 die Ausstellung „Per aspera ad astra“ im Foyer der Universitätsbibliothek Wien (Gesamtkonzeption und Gestaltung Wolfgang Rudolf KaINRATH) mit einer Präsentation der Sammlung der Universität Wien sowie Ausstellungen im Oberösterreichischen Landesmuseum (vgl. SPETA 1996) und im Radstädter Heimatmuseum.

Im Jahr 2007 wurde erstmals der Friedrich-Simony-Preis für Personen ausgeschrieben, die zur Förderung der Welterbe-Region und des Welterbe-Gedankens der Region Hallstatt - Dachstein - Salzkammergut beitragen.

Die Festveranstaltung des Instituts für Geographie und Regionalforschung der Universität Wien gemeinsam mit der Österreichischen Geographischen Gesellschaft am 22. November 2013, begleitet von einer Ausstellung in der Universitätsbibliothek Wien, kann ebenfalls in dieser Reihe genannt werden.

Zahlreiche geographische Objekte in den Alpen und ein Gletscher in der Arktis, nämlich auf der McClintock-Insel [Ostrov Mak-Klintoka] des Franz-Josef-Landes [Zemlja Franca-Josifa] oberhalb von Kap Brünn [Mys Brjun] auf etwa $80^{\circ} 30^{`}$ nördlicher Breite und $56^{\circ}$ östlicher Länge, wurden nach Simony benannt.

\section{Simony und die Geographische Gesellschaft}

Dem Sitzungsbericht der Geologischen Reichsanstalt vom 10. Februar 1852 kann die erste Anregung zur Gründung einer Geographischen Gesellschaft entnommen werden (S. 181-182). Bei der offiziellen Gründung am 21. September 1856 war Simony als Gründungsmitglied anwesend. Am 4. November 1856 wurde er zum Zweiten Sekretär in den Ausschuss gewählt. Er legte diese Funktion zwar kurz darauf wegen seiner vielfältigen Tätigkeiten zurück, war aber Ausschussmitglied von 1858 bis 1866 und von 1869 bis 1889, von 1863 bis 1964 außerdem Vizepräsident. Zwischen 1857 und 1885 verfasste SimONY 16 Veröffentlichungen in den „Mitteilungen der k.k. Geographischen Gesellschaft in Wien“. Eine ausführliche Darstellung der Beziehungen Simonys zur Geographischen Gesellschaft findet sich bei Kretschmer (1996a).

\section{Wissenschaftliche Forschungsfelder: gelebte Interdisziplinarität}

Fast genau 200 Jahre bevor Elisabeth Lichtenberger in der Festschrift zu ihrem 70. Geburtstag für diese herausragende Eigenschaft geehrt wurde, praktizierte SimONY von Beginn der universitären Geographie in Österreich an das Konzept der Geographie als Integrativwissenschaft.

Als Professor für die gesamte Geographie deckte er - bevor unter seinen Nachfolgern die „Zweitheilung der Geographie“ (in die Lehrkanzel für mathematisch-physikalische Geographie mit Albrecht Penck und die Lehrkanzel für historische Geographie mit Wilhelm TомAscheK) erfolgte - ein beachtliches Spektrum ab: Glaziologie, Geomorphologie, Limnologie, Kartographie, Landschaftsdarstellung, Didaktik und Lehrmittelherstellung, Meteorologie, Vegetationskunde waren die Teilgebiete, die er mit schriftlichen und graphischen Arbeiten sowie mit Fotografien bereicherte.

\subsection{Hochgebirgsforschung und Glaziologie}

Hubert NAGL (1996) gibt einen umfassenden Überblick über SIMONYs Hochgebirgsforschung. SimONY zeigt hier seine theoretische Stärke: Er formulierte aus morphologischen Beobachtungen Hypothesen über eiszeitliche morphologische Prozesse und wagte es, zur Theorie über vergangene Eiszeiten zu stehen, welche damals noch keineswegs anerkannte Tatsachen waren. 
Im Jahr 1846 beschrieb er Spuren vorgeschichtlicher Gletscherausdehnung, 1851 „Die Verbreitung erratischen Diluviums im Salzkammergut und deren Ähnlichkeit mit dem Moränenschutt der Dachsteingletscher“, 1869 publizierte er „Über die Urgesteinsablagerungen im Traunthale" (das Auffinden von Ennstal-Schottern im Trauntal) und erkannte schuttbeladene Eisströme als die Träger des Geschiebetransports, noch bevor 1875 der in der Fachwelt anerkannte Beweis der Vereisung durch Otto Martin Torell (1828-1900) gelang. SimONy beschrieb dies mit plastischen Worten:

„...Kolossale Eisströme, manche derselben mehrere tausend Fuß mächtig, und 10-20, ja noch mehr Meilen lang, drängen sich schuttbeladen durch die Thäler in das angrenzende Vorland hinaus.“ (aus dem Vortrag „Die Eiszeit der Diluvialperiode“, 1875)

Zwischen 1840 und 1890 kam Simony regelmäßig auf den Dachsteingletscher, um dessen Verhalten zu studieren und zu dokumentieren. Die Ergebnisse fasste er in seiner Schrift „Das Schwinden des Karlseisfeldes nach 50jährigen Beobachtungen und Aufnahmen" zusammen (Simony 1891).

Bereits 1863 stellte er in einem Vortrag Betrachtungen über die Schneegrenzen an (SIMONY 1864). Er vermerkte die unterschiedliche Höhenlage der Schneegrenze über Erdteile und Klimate hinweg und erkannte, dass die Schneegrenze nicht monokausal mit der Jahresisotherme zusammenhängt. Er nannte dazu als Beispiel zwei Gebirgsteile, die über die gleiche Wärmesumme verfügen, aber aufgrund der verschiedenen Niederschlagssummen unterschiedliche Schneegrenzhöhen haben. In seiner Beschreibung zum Wandtableau „Gletscherphänomene“ definierte er (um 1858) die Seitenmoränen knapp und unmissverständlich als



Quelle: Sammlung „Landschaftszeichnungen aus dem Salzkammergut“, Geologische Bundesanstalt

Abb. 2: „Das Innere einer Gletscherhöhle im Karlseisfeld im December 1842“. Aquarell, Version ohne Beschriftung 


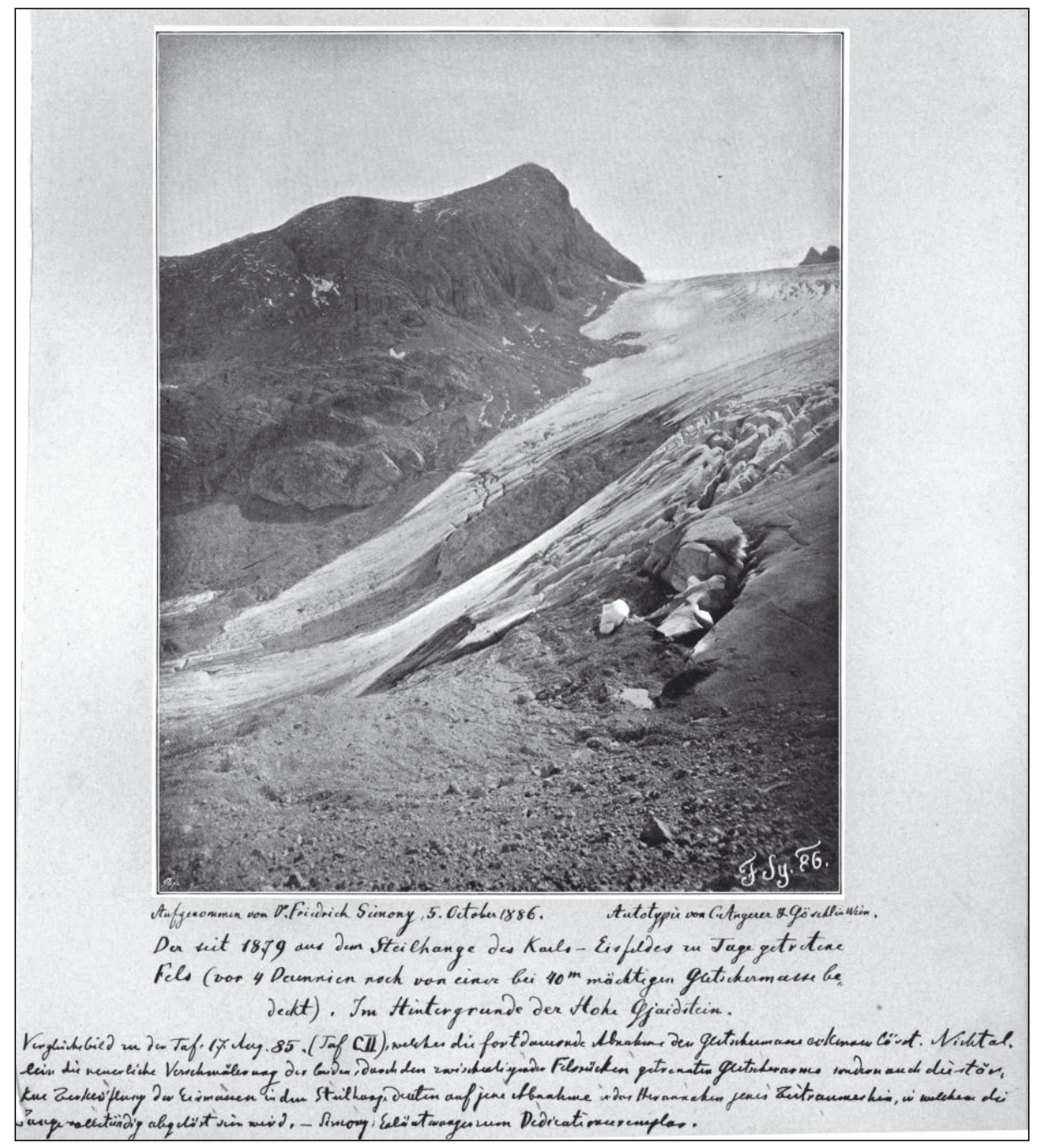

Quelle: Sammlung „Teilnachlass F. Simoney“, Universität Wien

Abb. 3: „Der Abfall des Karlseisfeldes zu seiner untersten Stufe im Oktober 1886 mit dem Hohen Gjaidstein im Hintergrunde“

Bildtext: „Aufgenommen von Dr. Friedrich Simony 5. October 1886. Autotypie von C. Angerer \& Göschl in Wien. Der seit 1879 aus dem Steilhange des Karls-Eisfeldes zu Tage getretene Fels (vor 4 Decennien noch von einer bis $40 \mathrm{~m}$ mächtigen Gletschermasse bedeckt). Im Hintergrunde der Hohe Gjaidstein. Vergleichsbild zu der Taf. 17. Aug. 85 (Taf CII), welches die fortdauernde Abnahme der Gletschermasse erkennen lässt. Nicht allein die neuerliche Verschmälerung der beiden durch den zwischenliegenden Felsrücken getrennten Gletscherarme sondern auch die stärkere Zerklïftung der Eismassen in dem Steilhang deuten auf jene Abnahme in den Herannahen jenes Zeitraumes hin, in welchem die Zunge vollständig abgelöst sein wird. - Simony, Erläuterungen zum Dedicationsexemplar.“ 
„langgestreckte Schuttstreifen, welche gewöhnlich erst unterhalb der Schnee-, wohl auch der Firngrenze, ..., sich bemerkbar machen...".

In seinem Vortrag „Eiszeitalter des Diluviums“ nennt er die wesentlichen Voraussetzungen für die Beziehungen zwischen Ufermoränen und Schneegrenze, welche später LichteNECKER (1938) bekannt machte.

Um 1858 beschreibt Simony zum ersten Mal das Ausapern der Seitenmoräne frühestens ab der Schneegrenze. Er beobachtete, dass eine Abnahme der Temperatur um $1{ }^{\circ} \mathrm{C}$ durchschnittlich einer Höhenzunahme von 180 m entsprach; für die freie Atmosphäre vermutete er Werte um 165 m. In einer modernen, aufwändigen wissenschaftlichen Arbeit zum Rückgang der Gletscher Graubündens wurden im Schnitt 170 m berechnet (MAISCH 1992).

Zwei bisher unveröffentlichte Werke SimONYs geben einen weiteren Einblick in die Fülle seiner Arbeiten. Abbildung 2 zeigt das Aquarell einer Gletscherhöhle, das zwei Jahre vor dem Bild ,,Karls-Eisfeld am Hohen Gjaidstein“, nämlich im Jahr 1842, entstanden ist. Abbildung 3 zeigt ein Blatt aus dem (unveröffentlichten) Sammelband „Die Textbilder der Schlusslieferung“ (Sammlung Universität Wien). Eine Phototypie des Bildes von ANGerer \& Göschl (Wien) wurde von SimONy eingeklebt und mit einer handschriftlichen Erläuterung versehen.

\subsection{Kartographische Arbeiten}

Einen Überblick über die Vielzahl an thematischen Karten und kartenverwandten Darstellungen gibt KRETSCHMER (1996b) mit der Abbildung und Beschreibung einiger Objekte aus den Veröffentlichungen SimonYs.

Im Folgenden sollen zwei einzigartige, unveröffentlichte Stücke vorgestellt werden: Abbildung 4 zeigt einen Ausschnitt aus der „Projection des Hallstätter Seebeckens“ - eine Darstellung wie sie 200 Jahre später in modernen Dokumentationen nach aufwändigen Echolotmessungen

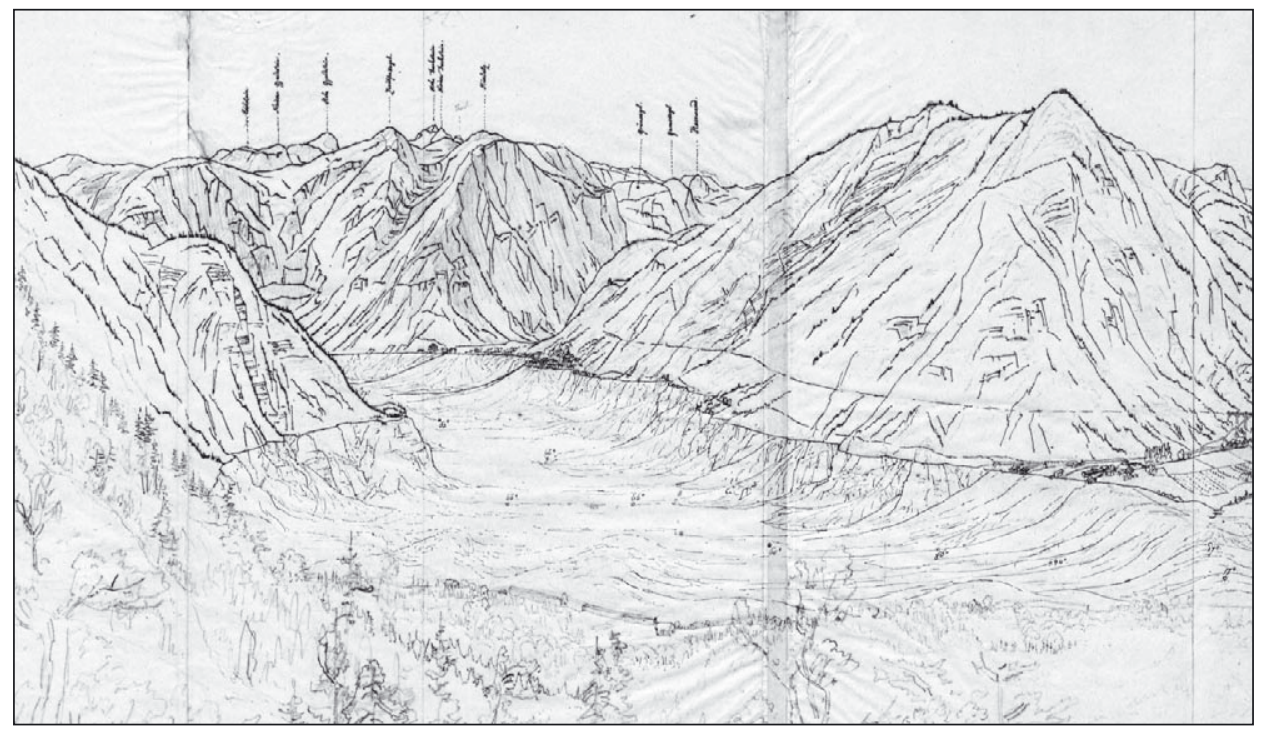

Quelle: Sammlung „Landschaftszeichnungen aus dem Salzkammergut“, Geologische Bundesanstalt

Abb. 4: „Projection des Hallstätter Seebeckens“, um 1846 


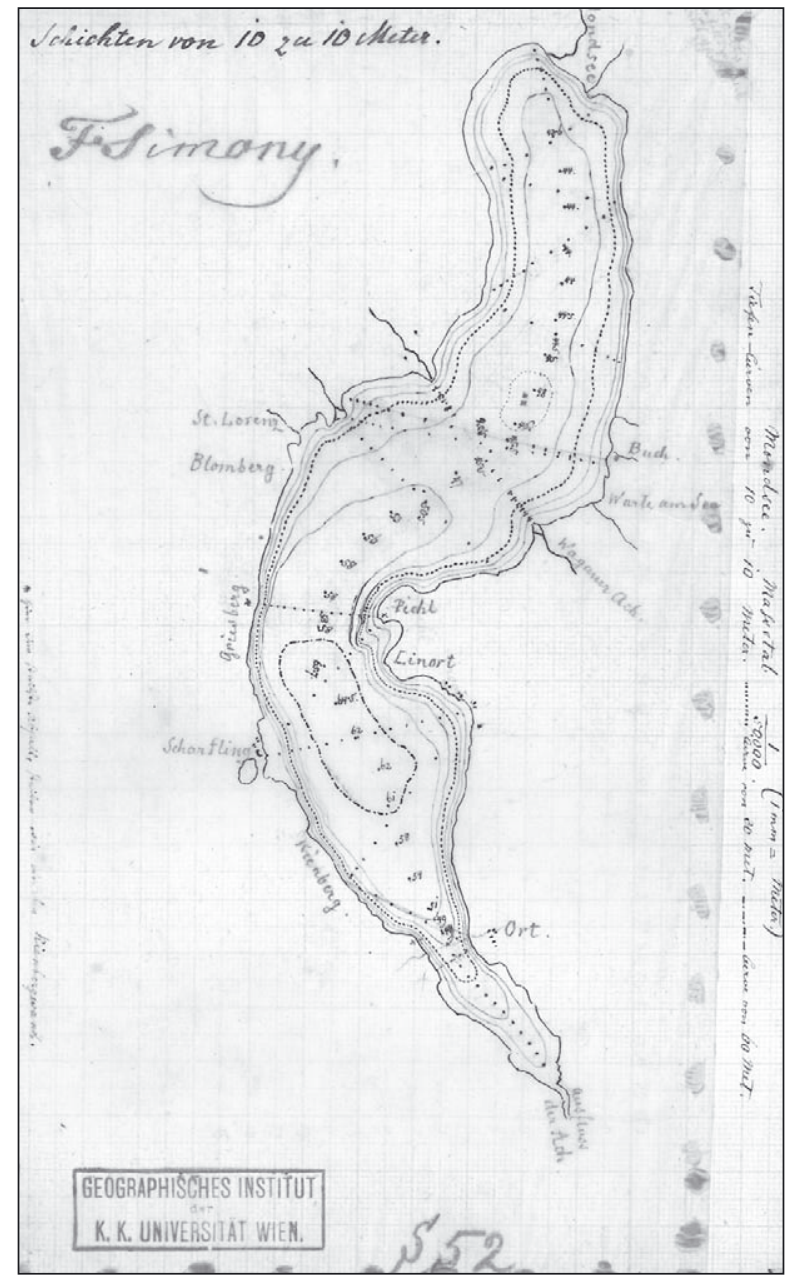

Quelle: Sammlung „Teilnachlass F. Simoney“, Universität Wien

Abb. 5: Mondsee 1:50.000 (Beschriftung am rechten Blattrand, vertikal zur Abbildung: „Mondsee. Maßstab 1:50 000 [i mm = Meter $]$ Tiefen-Curven 10 zu 10 Meter ...... Curve von 20 Met. .-.-- Curve von 60 Met.“)

und Weltraumbildern mit Computeranimation gemacht wird. Simony hat ein faszinierend gleichwertiges Ergebnis mit ungleich höherem Aufwand erreicht. In dieser Sammlung befindet sich auch eine Darstellung von Längs- und Querprofilen des Hallstätter Sees.

Eine offenbar dazugehörige Karte des Hallstätter Sees, „die Tiefenverhältnisse nach eigenhändigen Vermessungen“ durch SIмONY darstellend, besitzt die Sammlung der Universität Wien (vgl. Kainrath 2012, S. 77), ebenso die Bleistiftzeichnung „Mondsee 1:50.000“ (auf Millimeterpapier im Format 15,5 x 25,5 cm) (vgl. Abb. 5). Sie dürfte als Arbeitsgrundlage für Blatt X „Der Mondsee 1:25.000“ im Atlas der österreichischen Alpenseen (PENCK \& RichteR 1895-96) gedient haben. 
Eine weitere kartographische Kostbarkeit dieser Sammlung ist die „Schichtenkarte der österreichischen Alpen und des nördlich angrenzenden Gebietes (1865)“ - eine von SimONY angefertigte Studie mit nie angewandter, der Natur nachempfundener Farbskala mit der dunkelsten Farbe in der Mitte. Sie ist ausschnittsweise und mit Beschreibung ebenfalls 2012 erstmals veröffentlicht worden (vgl. KAINRATH 2012, S. 79).

\subsection{Vegetationskunde}

SimONYs vegetationskundliche Beiträge sind ebenfalls bei NAGL (1996) beschrieben. Seine bemerkenswerte Sammlung von 3.455 Herbarblättern wurde dem Naturhistorischen Museum in Wien zugeeignet (vgl. RiedL-Dorn 1996), wo sie bis dato leider so gut wie unauffindbar ist. Die Universität Wien besitzt ein Unikat, das am Geographischen Institut verblieben ist (Sammlung „Teilnachlass F. Simony“) und das 153 Präparate auf 78 Blättern enthält (drei Stück gesammelt 1925-26 in Brasilien von einer unbekannter Person und die Blätter 1-150 gesammelt von Simony, überwiegend im Jahr 1880).

\section{Graphische Arbeiten}

Den größten Anteil von SimONYs nicht-schriftlichen Arbeiten bilden graphische Arbeiten - von einfachen Bleistiftskizzen bis zu Gemälden im Wandtafelformat. Oft sind sie von hohem künstlerischen Wert oder bestechen durch ihre Detailtreue. Letzteres gilt besonders für Originaldarstellungen von Landschaften und für das Herausarbeiten charakteristischer Typenformen wie bei seinen „Charakterbildern“. Neben dem Naturhistorischen Museum haben die Österreichische Nationalbibliothek, die Universität Wien und die Geologische Bundesanstalt die wichtigsten und wertvollsten Bestände.

Viele hundert Skizzen, Zeichnungen, Aquarelle in präziser Ausführung kennzeichnen SimoNYs Arbeitstechnik vor der Anwendung der Fotografie. Dabei gestaltete er sowohl naturgetreue Abbildungen wie auch Typenbilder, welche der Natur nachempfundene, aber nicht reale Landschaften und Formen darstellen. Die Grundlage für letztere war vor allem sein außerordentliches didaktisches Talent. Ein prominentes Beispiel ist sein berühmtestes Werk „Gletscherphänomene“, welches auf zwei Weltausstellungen prämiert wurde und einen Großteil der an der Oberfläche sichtbaren glazialen und periglazialen Formen eines (alpinen) Zungengletschers darstellt (vgl. Simony ca. 1873).

\section{Dichtkunst}

Völlig kontrastierend zum wissenschaftlichen Schrifttum erscheinen SimONYs wenige (bislang bekannte) Versuche, sich literarisch zu betätigen. Zu einem Ritterstück des Hallstätter Volkstheaters (vgl. Penck 1898, S. 7) schrieb Simony das „Vorspiel zum Minnesänger“. Auch wenn die Spuren heute nicht mehr verfolgbar sind, eine Abschrift vom „Vorspiel“ ist seit damals im Geographischen Institut verblieben und befindet sich in der Sammlung der Universität Wien.

In der Wien-Bibliothek im Rathaus findet sich ein nicht minder kurioser Versuch in lyrischer Kunst: ein Gedicht mit dem Titel „Des Mannes letzte Fahrt“, abgedruckt in der Allgemeinen Theaterzeitung vom 5./6. Jänner 1844. Es wurde zufällig von O. PAUSCH (1997) in der 1971 in einem Antiquariat gefundenen „Denkschrift“ (vgl. SimONy 1851) wiederentdeckt. 


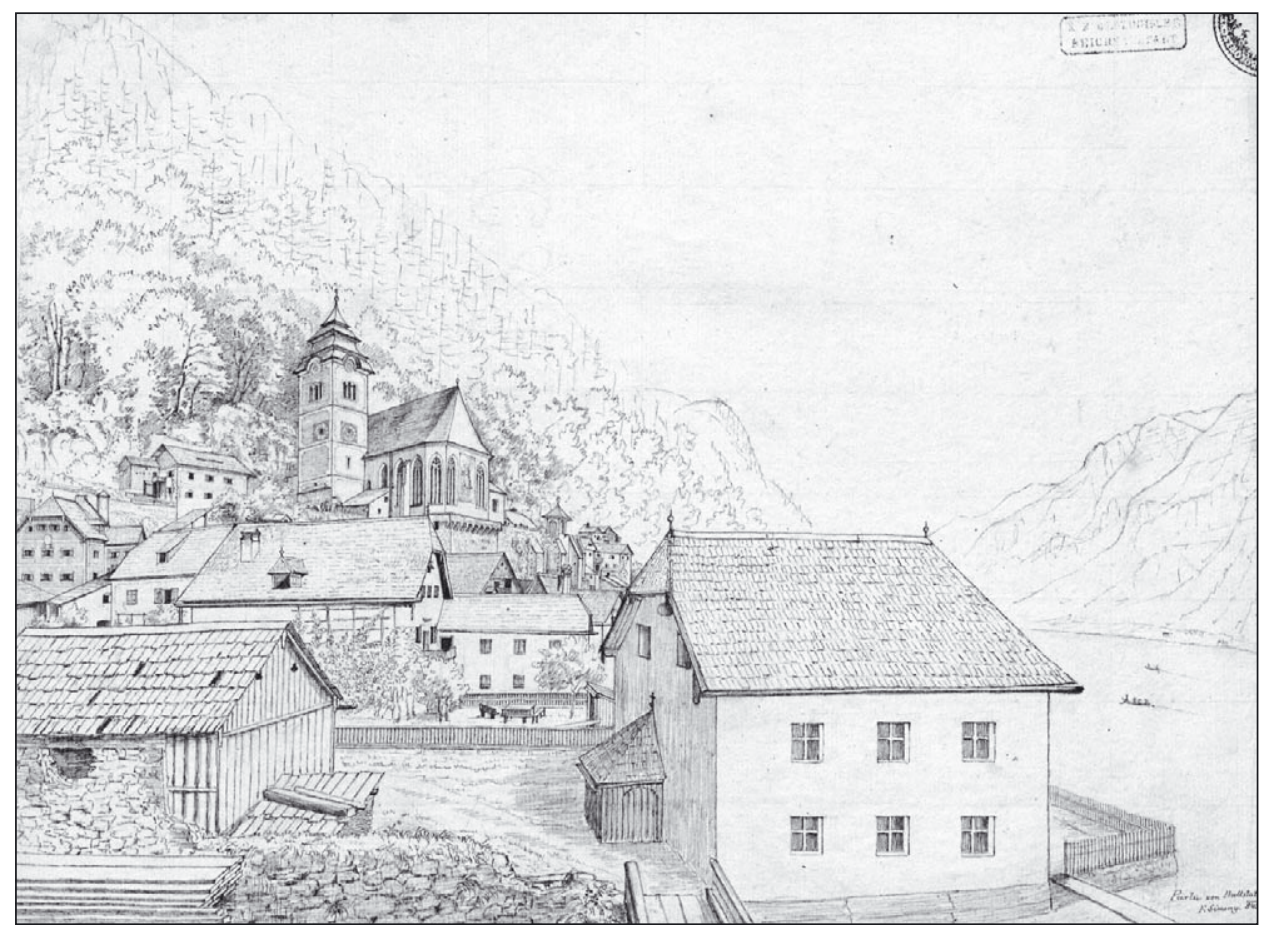

Quelle: Sammlung „Landschaftszeichnungen aus dem Salzkammergut“, Geologische Bundesanstalt

Abb. 6: „Partie von Hallstatt“ von Simony, 1843

\section{Sammlungen über Simony}

Es handelt sich um Ergänzungen zur Zusammenstellung der aufgefundenen Bestände bei KAINRATH 1996.

Archiv der Universität Wien: Personalakt Friedrich Simony, darin Ernennung zum Professor für Geographie an der Universität Wien (1851), Versetzung in den Ruhestand (1885), Korrespondenz zu dienstlichen Angelegenheiten; 118 Blatt Archivinformationssystem AIS via Homepage, Signatur PH PA 3173.

Haus-, Hof- und Staatsarchiv - Familienarchiv Braun: sechs Briefe von Dr. Friedrich SIMONY, 1886.01.21-1894.01.04, Familienarchiv Braun, privater Nachlass von Adolph Freiherr vON BRAUn 17.6.1818-4.3.1904 (Diplomat und Staatsmann, Kabinettsdirektor des Kaisers 1866-1899) HHStA SB NI Braun 9-1-109. Akten mit Briefwechsel.

Historisches Alpenarchiv der Alpenvereine Deutschland, Österreich und Südtirol http://www.historisches-alpenarchiv.de: elf Grafiken (Mehrfachexemplare bekannter Werke); diverse personenbezogene Akten wie Ehrungen, Todesanzeigen in Aktenbündeln; Denkschrift an Kaiser Ferdinand I. 1845/46 (Handschrift, undatiert, Geschenk v. Pausch O., Akz. Nr. 3065), enthält unter anderem zwei bislang unauffindbare Lithographien „Das Dachsteingebirge vom Hütteneck aus“ und „Das Karls-Eisfeld im Jahre 1840“, Abbildung und Volltext in Pausch 1997.

Metternich-Familienarchiv: Forschungsbericht, zwei Teile: „Physiognomie des Salzkammergutes“, „Das Dachsteingebirge“. 
Oberösterreichisches Landesarchiv: Manuskript „Die Familie Krackowizer“, Sign. S 662.

Österreichische Nationalbibliothek, Bildarchiv: sechs Porträts von Friedrich SIMONY, alle aus den biographischen Veröffentlichungen bekannt, sowie 17 Aufnahmen der Simonyhütte in den einzelnen Bauphasen.

Österreichische Nationalbibliothek, Handschriften- und Inkunabelnsammlung: Briefe (vgl. KLEMUn 1993 bezüglich Briefwechsel im Zusammenhang mit Simonys Tätigkeit in Kärnten); Sammlung August Miller-Aichholz; Berghaus, Heinrich [Verf.]; Ehrlich, Franz Karl [Verf.]; Haidinger, Wilhelm Karl von (1795-1871); Marschall-Burgholzhausen, August Friedrich [Verf.] (1804-?, Botaniker, Geologe); Morlot, Adolph von (1820-1867, Geologe, Archäologe; über 20 Briefe, ca. 100 Seiten, 1849-1854); Moro, Max von (1817-1899, Historiker, Jurist); LitTrow, Auguste von [Verf.] (1819-1890, Schriftstellerin); (Graf) ThUN UND HoHENSTEIn, Leo von (1811-1888); Vorlesungsmitschriften von BAUER, Adolf (1855-1919, Historiker). Simony, F. „Panorama des Schafberg“s nach der Natur gezeichnet und Seiner Kaiserlichen Hoheit Erzherzog Ludwig von Österreich in tiefster Ehrfurcht und Dankbarkeit gewidmet von Friedr. Simony“. Handschrift (Faltplan), zwölf Segmente, kolorierte Federzeichnung, 330 x 2844 mm; KARABACEK, Joseph von (1845-1918, Papierhistoriker, Kaiserlich-Königliche Hofbibliothek Wien).

Universität Wien: „Simony-Gedenksammlung“ mit vielen Volltexten (Biographie, Literatur, Werke), Bildern und Dokumenten im Langzeitarchivierungssystem Phaidra. - http://phaidra. univie.ac.at/o:298128

Universitätsbibliothek Wien: ,Vergleichende physische Geographie der Continente mit besonderer Berücksichtigung Europas“, Handschrift, 64 S., illustriert, gebunden. Signatur III300668.

Wiener Stadt- und Landesbibliothek: einige Briefe, unter anderen an Auguste von LittrowBischoff (aus deren Nachlass), Salzburg, 18.8.1873; an Friedrich Umlauft (aus dessen Nachlass), Wien, 24.11.1884; an Karl Weiß (aus dessen Nachlass), Wien, 2.1.1865. Handschrift „Des Mannes letzte Fahrt“ (o.J., 4 Seiten auf 1 Blatt, gef., Sign.: H.I.N.-85971).

\section{Literaturverzeichnis}

Allgemeine Theaterzeitung und Originalblatt für Kunst, Literatur, Musik, Mode und geselliges Leben, 5./6. Jänner 1844

Fischer H., Nagl H., Wohlschlägl H. (Hrsg.) (1996), Friedrich Simony-Gedenkband (= Geographischer Jahresbericht aus Österreich, 53). Wien, Institut für Geographie und Regionalforschung.

Historisches Alpenarchiv der Alpenvereine Deutschland, Österreich und Südtirol - http://www. historisches-alpenarchiv.de

KadletZ-SchöfFel H., Kadletz K. (2000), Metternich (1773-1859) und die Geowissenschaften. In: Berichte der Geologischen Bundesanstalt, 51, S. 51-52.

KainRath W. (1996), Friedrich Simony (1813-1896). Ein Lebensbild des Alpenforschers und ersten Ordinarius für Geographie an der Universität Wien. In: FISCHER H., NAGL H., Wohlschlägl H. (Hrsg.), Friedrich Simony-Gedenkband (= Geographischer Jahresbericht aus Österreich, 53), S. 9-22. Wien, Institut für Geographie und Regionalforschung.

KainRath W.R. (2012), Teilnachlass Friedrich Simony. In: Feigl C. (Hrsg.), Schaukästen der Wissenschaft. Die Sammlungen an der Universität Wien, S. 77-80. Wien, Böhlau.

Klemun M. (1993), Friedrich Simonys Beziehungen zu Kärnten - gezeigt anhand von Briefen. In: Carinthia II, 183/103.1993, 1, S. 7-25.

Kretschmer I. (1996a), Friedrich Simony (1813-1896) - zum 100. Todestag eines Gründungsmitgliedes. In: Mitteilungen der Österreichischen Geographischen Gesellschaft, 138, S. 275-280. 
Kretschmer I. (1996b), Kartographische Arbeiten Friedrich Simonys. In: Fischer H., Nagl H., WoHLSChlägl H. (Hrsg.), Friedrich Simony-Gedenkband (= Geographischer Jahresbericht aus Österreich, 53), S. 43-61. Wien, Institut für Geographie und Regionalforschung.

Lehr R. (1996), Ein Leben für den Dachstein. Friedrich Simony (1813-1896). In: Speta F. (Red.), Ein Leben für den Dachstein (= Kataloge des OÖ Landesmuseums, Neue Folge 103), S. 9-42. Linz, Land Oberösterreich, OÖ Landesmuseum.

LichteNECKER N. (1938), Die rezente und diluviale Schneegrenze in den Ostalpen. In: GötZINGER G. (Bearb.), Verhandlungen der III. Internationale Quartärkonferenz 1936, S. 141-147. Wien, Geologische Landesanstalt.

MaIsch M. (1992), Die Gletscher Graubündens. Rekonstruktion und Auswertung der Gletscher und deren Veränderungen seit dem Hochstand von 1850 im Gebiet der östlichen Schweizer Alpen (Bündnerland und angrenzende Gebiete) - Teil A: Grundlagen - Analysen - Ergebnisse. Universität Zürich, Habilitationsschrift.

N.N. (1852), Geologische Reichsanstalt, Sitzung am 10. Februar 1852. In: Jahrbuch der k.k. Geologischen Reichsanstalt, 3, S. 181-182.

NAGL H. (1996), Friedrich Simony als Hochgebirgsforscher und Glaziologe. Bewertung seines Werkes aus österreichischer und internationaler Sicht. Fischer H., NAGL H., WohlSCHLÄGL H. (Hrsg.), Friedrich Simony-Gedenkband (= Geographischer Jahresbericht aus Österreich, 53), S. 25-40. Wien, Institut für Geographie und Regionalforschung.

Pausch O. (1997), Neue Quellen zur Biographie Friedrich Simonys. In: Jahrbuch des Adalbert Stifter Institutes, 4, S. 94-121.

Penck A. (1898), Friedrich Simony. Leben und Wirken eines Alpenforschers. Ein Beitrag zur Geschichte der Geographie in Österreich (= Geographische Abhandlungen, 6, 3). Wien, Geographische Gesellschaft.

Penck A., Richter E. (1895-96), Atlas der österreichischen Alpenseen. Wien, Hölzel.

Ratzel F. (1886), Zur Kritik der natürlichen Schneegrenze. In: Mitteilungen der Deutschen Akademie der Naturforscher Leopoldina, XXII, 19, S. 19-24.

RIEdL-Dorn Ch. (1996), Die Sammlungen Friedrich Simonys am Naturhistorischen Museum in Wien. In: Speta F. (Red.), Ein Leben für den Dachstein (= Kataloge des OÖ Landesmuseums, Neue Folge 103), S. 199-266. Linz, Land Oberösterreich, OÖ Landesmuseum.

Sammlung „Landschaftszeichnungen aus dem Salzkammergut“ (1840-46), Geologische Bundesanstalt, 34 Bl. Zeichnungen und Aquarelle. Signatur A 00222-MA.

Sammlung „Teilnachlass Friedrich Simony“ der Universität Wien, Fachbereichsbibliothek für Geographie und Regionalforschung (Verwaltung)

Simony F. (1851), Denkschrift, 20.6.1853 [Brief an das Unterrichtsministerium. Inhalt: Plan über die Organisation des Unterrichts an der Universität]. Handschrift, abgedruckt bei Penck (1898), S. 14-20.

Simony F. (1864), Die Gletscher. In: Schriften des Vereins zur Verbreitung naturwissenschaftlicher Kenntnisse in Wien, 3, S. 335-378.

Simony F. (ca. 1873), Gletscher-Phänomene [Besonderer Abdruck aus den Berichten des Freiherrn von Helfert über die Ausstellung von Schul- und Unterrichtsgegenständen in Wien]. Wien, Artaria \& Comp, 2 B1., 4 B1. Fotografien.

Simony F. (1891), Das Schwinden des Karlseisfeldes nach 50jährigen Beobachtungen und Aufnahmen. In: Mitteilungen des Deutschen und Österreichischen Alpenvereins, Neue Folge 7, S. 43-48 und 61-65.

SPETA F. (Red.) (1996), Ein Leben für den Dachstein (= Kataloge des OÖ Landesmuseums, Neue Folge 103). Linz, Land Oberösterreich, OÖ Landesmuseum.

Torell O. (1875), Gletscherschliffe von Rüdersdorf. In: Zeitschrift der deutschen geologischen Gesellschaft, 27, S. 961-962. 
Mitteilungen der Österreichischen Geographischen Gesellschaft, 155. Jg. (Jahresband), Wien 2013, S. 317-318

\title{
26. Internationale Kartographische Konferenz
}

\section{Dresden, 25. bis 30. August 2013}

\author{
Karel KRIZ und Alexander PUCHER, beide Wien*
}

„From Pole to Pole“ war das Motto der diesjährigen Internationalen Kartographischen Konferenz, die in Dresden vom 25. bis 30. August 2013 abgehalten wurde. Rund 1.350 Teilnehmer aus 82 Ländern konnten heuer aus über 600 Paper- und Poster-Präsentationen wieder ihre maßgeschneiderte kartographische Woche zusammenstellen. Unter der Leitung von Prof. Dr. Manfred Buchroithner und seinem Team wurde ein fachlich ebenso interessantes wie innovatives Programm geboten, welches noch zusätzlich vom sommerlichen Wetter gekrönt wurde. Die insgesamt 68 österreichischen Teilnehmer, die der Zahl nach die viertstärkste Nation bildeten, konnten sich mit zahlreichen Fachpräsentationen im Rahmen der Tagung hervorragend positionieren oder durch die aktive Teilnahme an Kommissionssitzungen und sonstigen Veranstaltungen deutliche Akzente setzen. Die Abhandlungen (Proceedings) der Fachpräsentationen, die auf einem USB-Stick sowie online ${ }^{1)}$ bereitgestellt wurden, enthalten sämtliche Beiträge der Konferenz, darunter auch jene der österreichischen Teilnehmer.

Die Technische Universität (TU) Wien war mit mehreren aktiven Teilnehmern vertreten. S. KletTNER et al. hielten einen Vortrag zum Thema „Acquisition and Cartographic Applications of Subjective Geodata“. E. ÖZERDEM et al. trugen zur Thematik „Evaluating the suitability of Web 2.0 technologies for online atlas access interfaces“ vor. „Barriers for contributing to VGI projects" war der Titel des Beitrags von M. SснмiDT et al. Außerdem referierte sie zum Thema "Challenges in creating web base maps from distributed datasets“. E. SimonNÉ-DombóvÁRI et al. präsentierten neueste Erkenntnisse im Rahmen der Ausführungen zu „Austrian-Hungarian survey on chernoff faces: an alternative method of representation in school cartography". H. HuANG et al. widmeten sich dem Themenkomplex der Location Based Services im Vortrag „Learning from location history for context-aware location recommendation in LBS“. M. JOBST, der außer die TU auch das Bundesamt für Eich- und Vermessungswesen repräsentierte, sprach zum Thema „Backend architectures for modern cartography“. Den Nutzern von frei verfügbaren Geoinformationen widmete J. BeHRENs seinen Beitrag „Users and Uses of OpenStreetMap“.

Neben der TU Wien trugen auch mehrere Vertreter der Universität Wien zum Vortragsprogramm bei. A. Pucher et al. referierten zum Thema „Geographic Space in Museums - Evaluation and Representation of Geographic Space within a Numismatic Exhibition“. F. Hruby et al., die neben der Universität Wien auch die Universität Guadalajara, Mexiko, vertraten, besprachen neue Erkenntnisse zur Berechnung von Distanzen auf unterschiedlichen Grundlagen in ihrem Vortrag „Maps vs. Globes - Distance Estimation on Flat and Spherical Displays“. „Utilizing a Mobile Smartphone Application in a Mountainous Environment” war der Titel des Beitrags

\footnotetext{
* Ass.-Prof. Mag. Dr. Karel KrIZ, Mag. Dr. Alexander Pucher, beide Institut für Geographie und Regionalforschung, Universität Wien, Universitätsstraße 7, A-1010 Wien; E-Mail: karel.kriz@univie.ac.at, alexander.pucher@univie.ac.at, http://www.univie.ac.at/cartography

1) http://icaci.org/files/documents/ICC_proceedings/ICC2013/
} 
von K. Kriz und B. HaJeK. Einen inhaltlichen Bogen zu kulturhistorischen Themen konnte M. BREIER in seiner Präsentation ,The Way is the Goal - Modelling of historical roads" spannen.

F. Leberl et al. (Institute for Computer Graphics and Vision, Technische Universität Graz) lieferten in ihrer Key-Note-Präsentation „The Virtual Centimeter World Model“ einen spannenden Blick in die Zukunft. Details zu neuen topographischen Karten im Rahmen der Österreichischen Landesverteidigung präsentierten R. Ditz et al. (Militärgeographisches Institut des Bundesministeriums für Landesverteidigung und Sport) in ihrem Beitrag „The new civil-military topographic maps of Austria“. Darüber hinaus hielt Diтz einen Vortrag zum Thema „The production of orienteering maps in Austria“. „Lunar and planetary globes in the holdings of the Austrian National Library's Globe Museum" war der Inhalt der Präsentation von J. MoKre (Österreichische Nationalbibliothek). B. HoFER (Universität Salzburg) trug das Thema „Cartography and Geoinformatics in Salzburg, Austria“ vor. Im Vortrag „ThermoMap - An Open Source Web Mapping Solution for Displaying Superficial Geothermic Resources“ präsentierten L. MORPER-Busch et al. (Universität Salzburg) aktuelle Ergebnisse. Neueste Forschungsergebnisse zum Rückgang der Gletscherstände wurden von V. KaufManN (Technische Universität Graz) in der Präsentation „Documentation of the glacier retreat in the eastern part of the Granatspitz Mountains (Austrian Alps) using aerial photographs for the time period 2003-2009“ dargelegt. Dem Thema VGI widmete L. SeE (International Institute for Applied Systems Analysis, Ecosystems Services and Management, Laxenburg) den Vortrag „Managing and Acquiring Volunteered Geographic Information“.

Einen beachtlichen Beitrag zur starken Präsenz der Österreicher leistete auch eine kartographische Fachexkursion der Universität Wien, Institut für Geographie und Regionalforschung, unter der Leitung von Wolfgang KaINZ. Den engagierten Studierenden der Geographie und Kartographie wurde einerseits die Gelegenheit geboten, eine internationale Fachtagung hautnah zu erleben, andererseits hatten sie die Aufgabe, ausgewählte Vortagsblöcke, die zu einem bestimmten Themenbereich zu verfolgen waren, kritisch unter die Lupe zu nehmen und zu analysieren.

Neben dem wissenschaftlichen Programm fand auch diesmal die bereits traditionelle internationale kartographische Ausstellung statt. Sie umfasste über 400 Exponate aus 35 Ländern. Die Ausstellungsobjekte wurden in fünf Hauptgruppen unterteilt: Karten, Atlanten, digitale Produkte, Bildungsbereich und Sonstiges. Österreich hat sich - wie stets - beteiligt, diesmal mit sechs Objekten. Darüber hinaus wurden mehrere handgezeichnete Karten österreichischer Schulkinder zur Barbara Petchenik Children's Map Competition 2013 eingereicht und ausgestellt. Hierbei ist besonders der dritte Platz in der Kategorie 9 bis 12 Jahre von Florian Gruber und Lukas Schostal sowie ihrer Lehrerin, Frau Mag. Erna Daublebsky, aus dem Polgargymnasium (Wien) zu erwähnen.

Im Rahmen der Abschlussfeier wurden vier Persönlichkeiten geehrt: Fraser TAYLOR aus Kanada erhielt nicht nur für seine langjährige Tätigkeit als Präsident der Internationalen Kartographischen Vereinigung (International Cartographic Association, ICA) die „Carl Mannerfelt Gold Medal“; Kirsi Virrantaus aus Finnland sowie Milan KonecnÝ aus Tschechien wurden die Auszeichnung „ICA Honorary Fellowship“ verliehen. Last but not least erhielt Manfred BuCHRoITHNER in Anerkennung seiner Dienste für die ICA das „Diploma for outstanding services to ICA" überreicht.

Weitere Informationen zur Tagung in Dresden sowie zur kommenden Tagung in Rio de Janeiro im Jahr 2015 können der Homepage der ICA (http://icaci.org) bzw. der Homepage der jeweiligen Veranstalter (http://www.icc2013.org/; http://www.icc2015.org/) entnommen werden. 
Mitteilungen der Österreichischen Geographischen Gesellschaft, 155. Jg. (Jahresband), Wien 2013, S. 319-320

\title{
4. EUGEO-KONGRESS
}

\section{Rom, 5. bis 7. September 2013}

\author{
Robert MusIL, Wien* \\ mit 1 Abb. im Text
}

Die EUGEO, die European Society for Geography, hat mit ihrer vierten Tagung ein kräftiges Lebenszeichen von sich gegeben. Der EUGEO-Kongress unter dem Motto „Europe, what's next? Changing geographies and geographies of change“" wurde vom 5. bis 7. September 2013 in Rom [Roma], an der Universität La Sapienza abgehalten. An den drei Kongresstagen fanden 97 Sitzungen statt, die durch eine starke Beteiligung italienischer Wissenschafter gekennzeichnet waren, was allerdings keineswegs eine thematische oder räumliche Einengung bedeutete. Immerhin kamen 56\% der Teilnehmer aus dem Ausland.

Trotz der breiten thematischen Streuung haben sich gewisse Schwerpunkte feststellen lassen. So standen erstens - dem Selbstverständnis der EUGEO entsprechend - europäische Themen im Mittelpunkt zahlreicher Sitzungen: Territorial- und Kohäsionspolitik, Grenzziehungen im Inneren und nach Außen, die Governance europäischer Städte und natürlich der räumliche Einfluss der gegenwärtigen Wirtschaftskrise, um die vielleicht wichtigsten zu benennen. Als weiterer Schwerpunkt hat sich der Bereich Umwelt/Naturraum/Landschaft abgezeichnet, der in den Sitzungen aus einer globalen (Global Change, Agrarpolitik, agrarische Wertschöpfungsketten, globaler Umweltwandel, Perzeption von Naturkatastrophen) sowie einer regionalen Perspektive (Landwirtschaft in verstädterten Räumen, Governance einer nachhaltigen Flächennutzung) intensiv behandelt wurde. Der dritte Schwerpunkt umfasste methodische Themen wie etwa der Kartographie, der Anwendungsmöglichkeiten von Big Data sowie die paradigmatische Entwicklung geographischer (Sub-)Disziplinen (Historische Geographie, Literatur und Geographie, Trends der Wirtschaftsgeographie).

Neben dem wissenschaftlichen Programm beeindruckte der Kongress mit atmosphärisch eindrucksvollen Standorten des Rahmenprogramms. Die Eröffnungsveranstaltung fand am Kapitol [Campidoglio] ganz in der Nähe des Forum Romanum [Foro Romano] statt (vgl. Abb. 1); eine Plenarsitzung in der Villa Celimontana, dem Sitz der Italienischen Geographischen Gesellschaft, das anschließende Dinner mit Konzert im Kolosseum [Colosseo].

Auch die Vorträge der vier Plenarveranstaltungen mit je zwei Vorträgen spiegeln die Vielfalt der EUGEO 2013 wider. Ron Boschma sprach zur Evolutionären Wirtschaftsgeographie, Petros Petsimeris über urbane Transformationen Europas, Peter Mehlbye über Evidenzen aus dem ESPON-Datenpool, Gyula HorvÁTH über die Dezentralisierung der Wissenschaft im östlichen Europa. Neben den unterschiedlichen Inhalten zeigten diese Vortragsduos - wie auch

\footnotetext{
* Mag. Dr. Robert MusIL, Institut für Stadt- und Regionalforschung, Österreichische Akademie der Wissenschaften, Postgasse 7/4/2,A-1010 Wien; E-Mail: robert.musil@oeaw.ac.at, http://www.oeaw.ac.at/ isr
} 
einige andere - auf beeindruckende Weise die Unterschiede der verschiedenen europäischen Wissenschaftskulturen. Somit war dieser Kongress von einem faszinierenden europäischen Kosmopolitismus geprägt, der andere „globale“ Kongresse in den Schatten stellt.

Der fünfte EUGEO-Kongress im Jahr 2015 wurde an Budapest vergeben.

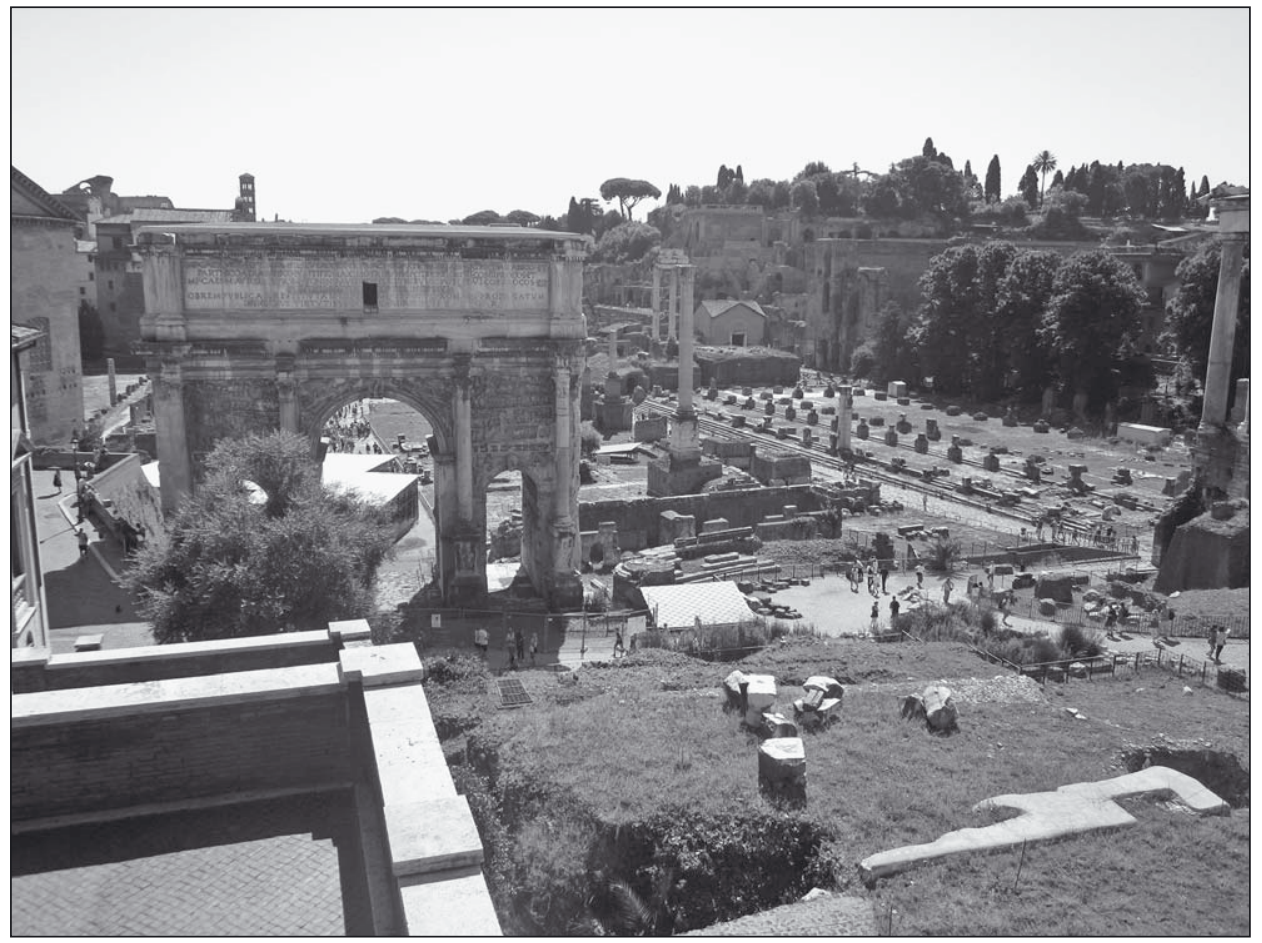

Abb. 1: Das Forum Romanum vom Kapitol aus (Foto: Peter JoRDAN) 
Mitteilungen der Österreichischen Geographischen Gesellschaft,

155. Jg. (Jahresband), Wien 2013, S. 321-326

\title{
Der 58. Deutsche Geographentag 2013 in Passau - Ein grosser Kongress in EINER KLEINEN STADT
}

\author{
Peter Alexander RuMPOLT, Wien*
}

mit 5 Abb. im Text

\section{INHALT}

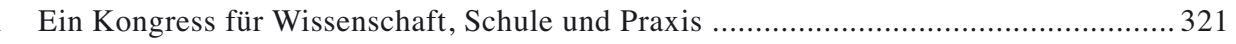

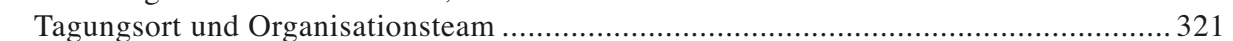

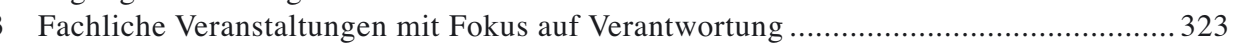

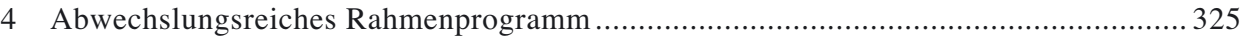

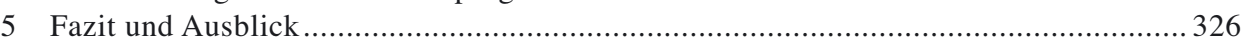

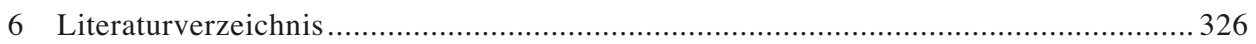

\section{Ein Kongress für Wissenschaft, Schule und Praxis}

Vier Jahre nach dem 57. Deutschen Geographentag, jenem Ende September 2009 in Wien, stand vom 2. bis 8. Oktober 2013 die nächste Ausgabe dieser für die deutschsprachige Geographie zentralen Tagung am Programm. Austragungsort war erstmals die kreisfreie Stadt Passau in Niederbayern mit ihrer 1978 eröffneten Universität.

Der insgesamt 58. Deutsche Geographentag war gleichzeitig der dritte in Folge, welcher als ein großer Kongress für Wissenschaft, Schule und Praxis abgehalten wurde. Man blieb somit jenem Konzept treu, das sich 2007 in Bayreuth und 2009 in Wien bewährt hatte und welches darin besteht, eine gemeinsame Tagung für Hochschul-, Schul- und Berufsgeographinnen und -geographen zu veranstalten. Dies kann sich auf den Austausch und Dialog innerhalb der Geographie förderlich auswirken (vgl. MusIL 2009, S. 315). Im Jahr 2011 hatte im Übrigen kein Deutscher Geographentag stattgefunden. Im Jahr 2012 war Köln der Austragungsort des Weltkongresses der Geographie (International Geographical Congress; vgl. Nissel \& Embleton-Hamann 2012).

\section{Tagungsort und Organisationsteam}

Die in unmittelbarer Nachbarschaft zu (Ober-)Österreich und unweit des Dreiländerecks zwischen Deutschland, Österreich und Tschechien am Zusammenfluss von Inn, Donau und Ilz gelegene niederbayerische Grenzstadt Passau stellte für den 58. Deutschen Geographentag einen

* Mag. Peter Alexander Rumpolt, Bundesanstalt Statistik Österreich, Guglgasse 13, A-1110 Wien, und Institut für Geographie und Regionalforschung, Unversität Wien, Universitätsstraße 7, A-1010 Wien; E-Mail: peter.rumpolt@statistik.gv.at, peter.rumpolt@univie.ac.at,http://www.statistik.at, http://geo graphie.univie.ac.at 


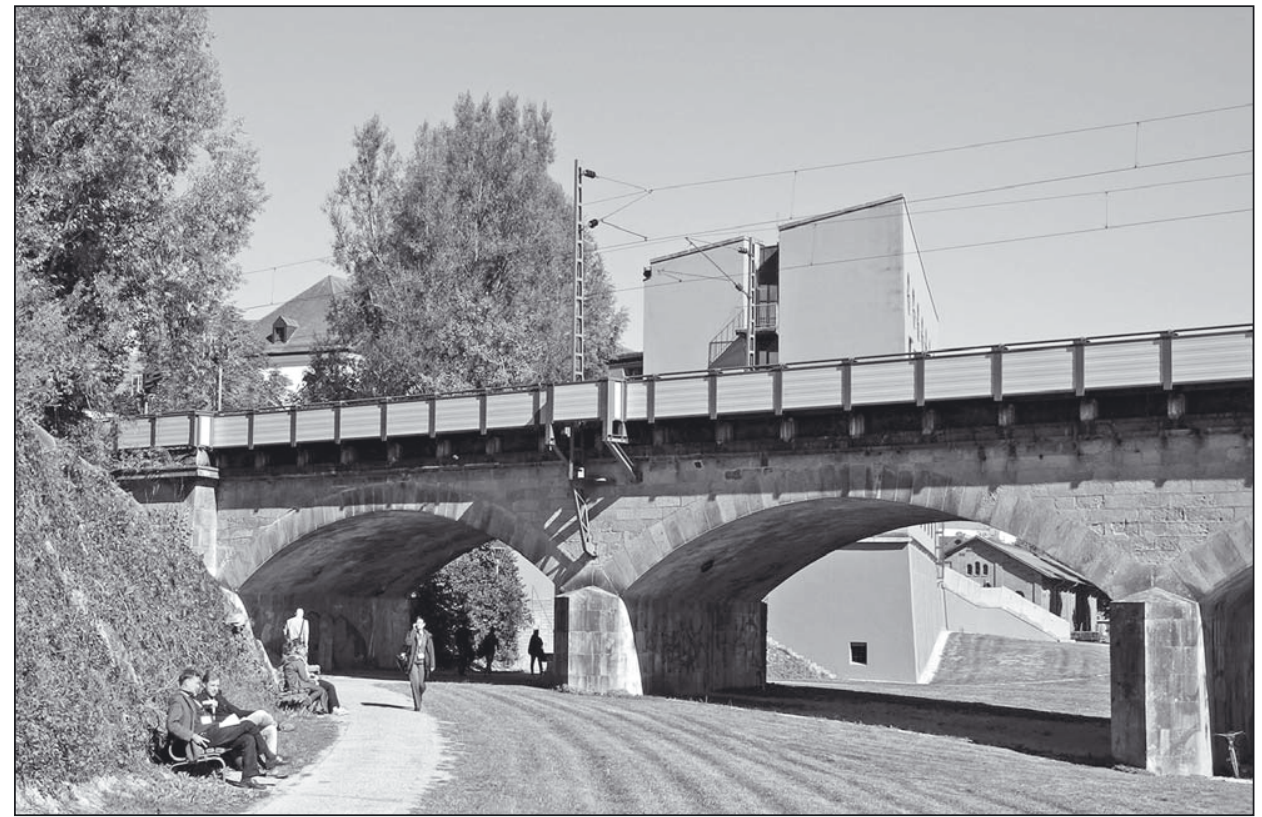

Abb. 1: Während des Geographentages 2013 am Campusgelände der Universität Passau (Foto: P.A. Rumpolt)

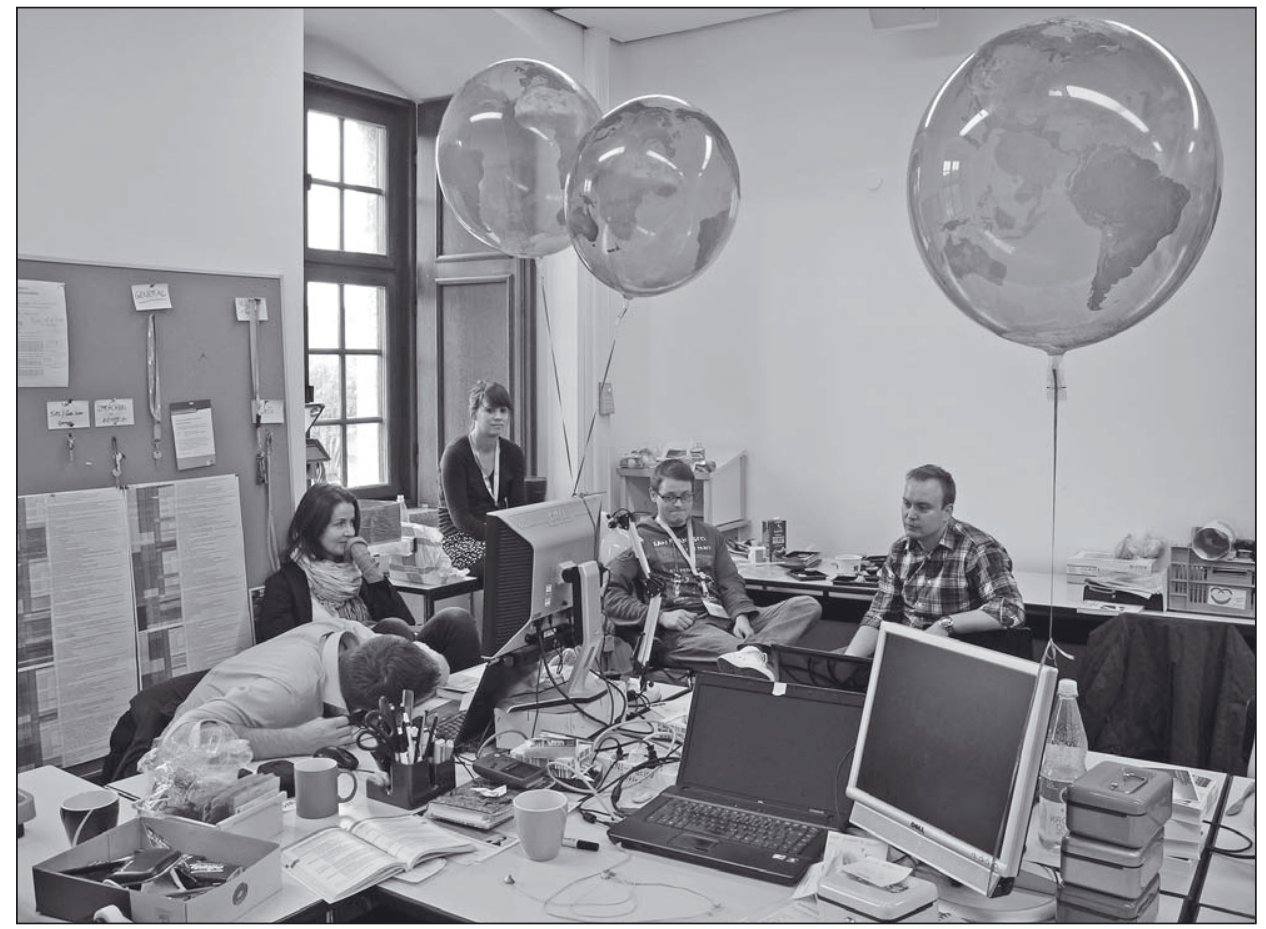

Abb. 2: Ein Blick in das Back-Office des Geographentages in Passau (Foto: P. A. Rumpolt) 
charmanten Tagungsort dar. Auch das Tagungsgelände im engeren Sinn, der direkt am orographisch linken Ufer des Inn gelegene Campus der Universität Passau mit seinen architektonisch vielfältigen Gebäuden, wies ein angenehmes und einladendes Ambiente auf (vgl. Abb. 1).

Im Gegensatz zum unmittelbaren „Vorgänger“ sowie „Nachfolger“ bei der Ausrichtung Deutscher Geographentage, den Millionenstädten Wien und Berlin, kann Passau mit seinen rund 50.000 Einwohnern als vergleichsweise kleine Universitätsstadt bezeichnet werden. Demnach und unter Bedachtnahme auf die Tatsache, dass auch der Fachbereich Geographie der Universität Passau eher zu den kleineren im deutschen Sprachraum zählt, ist eine erfolgreiche Ausrichtung einer solchen wissenschaftlichen Großveranstaltung umso bemerkenswerter.

Voraussetzung dafür scheint ein bestmögliches Zusammenspiel des gesamten Organisationsteams zu sein. Dieses Team stand unter der Leitung von Kongresskoordinator Michael HEUbERGER - der (nach Wien 2009) bereits zum zweiten Mal einen Geographentag federführend organisierte - und bestand darüber hinaus aus den weiteren Mitgliedern des eigentlichen Geographentagsmanagements, den Mitgliedern des Ortsausschusses Passau (Dieter AnHuf, Werner GAMERITH und Ernst StRUCK) und weiteren Mitarbeiterinnen und Mitarbeitern des Fachbereichs Geographie der Universität Passau sowie den im Bereich von Universität und Stadt Passau für entsprechende Verwaltungsagenden zuständigen Personen und „last, but not least“ aus einer großen Zahl an Studierenden. Diese überwiegend weiblichen Studierenden - die blau gewandete "Crew" - erwiesen sich als jederzeit freundlich und hilfsbereit. In Passau hat man somit 2013 tatsächlich bewiesen, ,[...] dass - mit dem Rückhalt der Universität, einer engen, kollegialen Zusammenarbeit der Geographinnen und Geographen vor Ort und einem feinmaschigen Netz institutionalisierter und fachlicher Kontakte - auch ein kleiner Standort einen großen Wissenschaftskongress gestalten und beherbergen kann“ (ANHUf et al. 2013, S. 1). Die Bedeutung des Standorts Passau innerhalb der deutschsprachigen Geographie fand wohl auch darin ihren Ausdruck, dass Werner GAMERITH während des 58. Deutschen Geographentages zum nächsten Präsidenten der Deutschen Gesellschaft für Geographie (DGfG) gewählt wurde und somit in dieser Funktion mit 1. Jänner 2014 die Nachfolge von Hans-Rudolf BorK antreten wird.

\section{Fachliche Veranstaltungen mit Fokus auf Verantwortung}

Die thematische Fokussierung des Deutschen Geographentages 2013 wird anhand des Tagungsmottos „VerANTWORTen - Herausforderungen der Geographie“ deutlich. Diesem Motto folgend war der Geographentag in Passau ,[...] der wichtigen Frage nach der gesellschaftspolitischen Verantwortung der Wissenschaftsdisziplin, des Berufsfelds und des Schulfachs Geographie“ (ANHUf et al. 2013, S. 1) gewidmet. In Anknüpfung daran stand auch das Zusammenspiel der wissenschaftlichen Disziplin Geographie mit den Medien und der Öffentlichkeit im Blickpunkt. In diesem Kontext fanden auch mehrere Leitthemensitzungen statt, unter anderem eine zum Thema „Verantwortung übernehmen: Geographie in der Öffentlichkeit“, im Rahmen derer unter anderem auch ein Vortrag von Werner GAMERITH mit dem Titel „Geographische Gesellschaften und geographische Öffentlichkeit - vom kolonialen Relikt zum zeitgemäßen Instrument der Wissenschaftskommunikation“ am Programm stand und diesbezüglich interessante Überlegungen darbot.

Dass „Verantworten“ auch „Antwort“ impliziert, konnte auch der in Verbindung mit dem „Drei-Flüsse-Logo“ des Passauer Geographentages verwendeten Schreibweise des Tagungsmottos (vgl. Abb. 3) offensichtlich sowie dem Namen des an den vier Kerntagen des Kongresses (3. bis 6. Oktober 2013) täglich aufgelegten zweiseitigen Newsletters (vgl. Abb. 4) implizit entnommen werden. Darüber hinaus wurde darauf beispielsweise auch von dem an der Universität Wien 

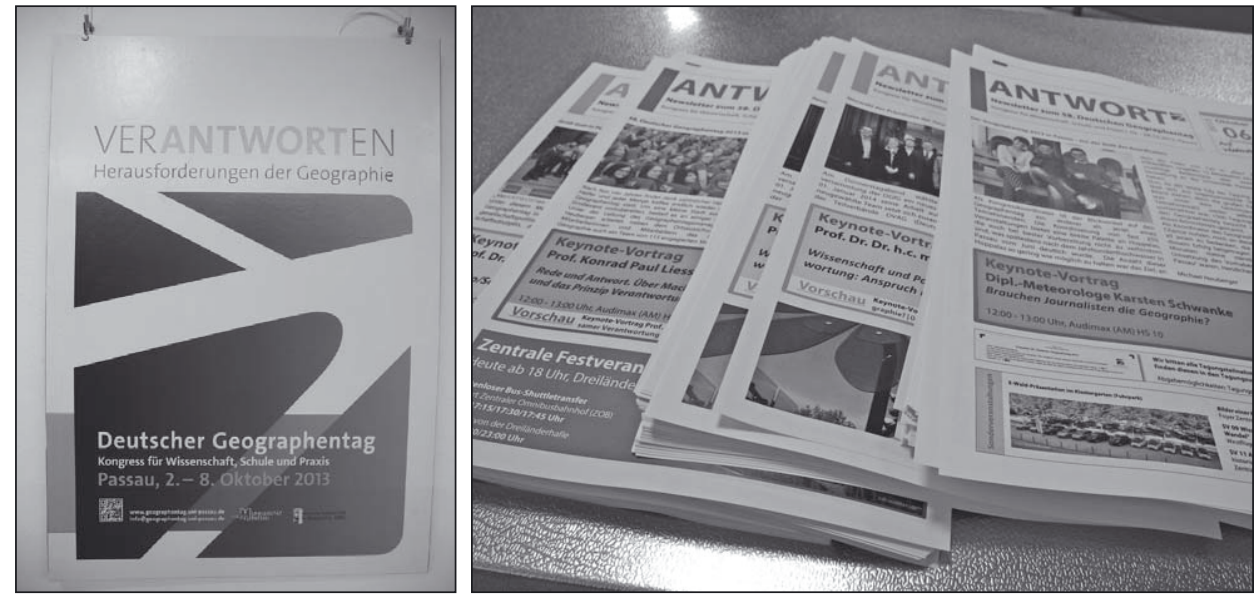

Abb. 3: links: Motto und Logo des Geographentages in Passau auf einem Ankündigungsplakat

Abb. 4: rechts: Die vier Ausgaben des Geographentags-Newsletters „Antwort“ (Fotos: P.A. Rumpolt)

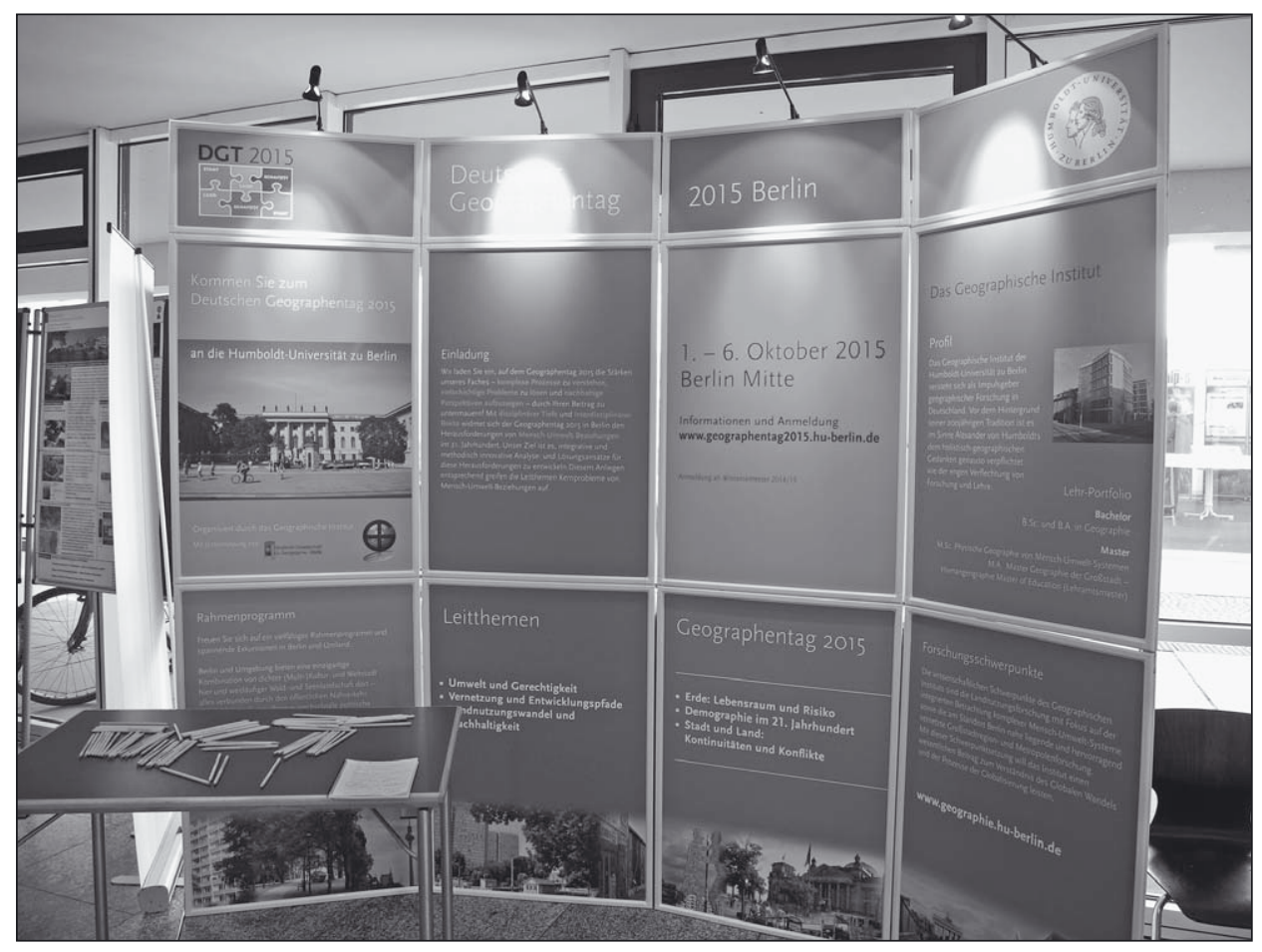

Abb. 5: Präsentationsstand des nächsten Deutschen Geographentages 2015 in Berlin (Foto: P.A. Rumpolt) 
forschenden und lehrenden Philosophen Konrad Paul Liessmann in seinem Keynote-Vortrag mit dem Titel „Rede und Antwort. Über Macht, Moral und das Prinzip Verantwortung“ explizit und eindrucksvoll Bezug genommen. ,Verantwortung zu erfüllen heißt Antworten zu geben auf die großen Fragen der Gegenwart und Zukunft [...]" hielten Dieter Anhuf et al. (2013, S. 1) bereits in der ersten Ausgabe des Newsletters zum Geographentag fest.

Zusätzlich zu vier Keynote-Vorträgen und 18 Leitthemensitzungen standen beispielsweise auch Sonderveranstaltungen im Zeichen der inhaltlichen Schwerpunktsetzung: Dabei stellten die Präsentation von Elektromobilität unter dem Motto „Mobilität verANTWORTen“ sowie die Möglichkeit zu deren praktischer Erprobung im städtischen und ländlichen Alltag zweifellos einen wertvollen Mehrwert und auch eine interessante Erfahrung dar. Im Rahmen des Geographentages in Passau wurde Verantwortung also auch sehr umsetzungsorientiert gelebt.

Aber auch abseits des speziellen Tagungsmottos wurde eine große Palette an fachspezifischen Veranstaltungen angeboten, wobei die Fachsitzungen, speziell im Bereich der Anthropo- oder Humangeographie, eindeutig das Gros ausmachten. Fachlich ergänzt wurden die Keynote-Vorträge, Leitthemen- und Fachsitzungen durch Exkursionen und Arbeitskreissitzungen sowie Veranstaltungen des Jungen Geographentages, Workshops, Podiumsdiskussionen, Posterpräsentationen, die Fach- und Verlagsausstellung und eben auch Sonderveranstaltungen. Während im Rahmen des Programms des Jungen Geographentages unter anderem Einblicke in abgeschlossene sowie laufende Forschungsprojekte und Abschlussarbeiten gewährt wurden, umfasste der Bereich der Sonderveranstaltungen unter anderem auch Ausstellungen. Dazu zählte die wissenschaftliche Posterausstellung „Alpen - Lebensraum im Wandel“ der Österreichischen Geographischen Gesellschaft (vgl. Rumpolt 2013, S. 3, sowie vor allem auch Rumpolt in diesem Band) ebenso wie eine anlassbezogen um eine Fotodokumentation des Hochwasserereignisses in Passau Anfang Juni 2013 erweiterte Ausstellung über Stadt und Region Passau in historischen Ansichten.

\section{Abwechslungsreiches Rahmenprogramm}

Den Mittelpunkt des Rahmenprogramms und gleichzeitig auch die Verbindung zum fachlichen Programm bildete eine zentrale Festveranstaltung, welche das System vergangener Geographentage mit jeweils einer Eröffnungs- und einer Schlussveranstaltung ablöste. Diese am 4. Oktober 2013 in der Dreiländerhalle abgehaltene zentrale Festveranstaltung umfasste Ansprachen von Fachvertretern und Politikern sowie Auszeichnungen und Preisverleihungen ebenso wie eine nicht zu kurz geratene musikalische Gestaltung durch ein Ensemble aus Oberammergau und einen anschließenden kulinarischen Ausklang des Abends.

Während der an diesem Abend mehrfach, auch von politischer Seite vorgebrachte Hinweis auf die zentrale Bedeutung der Geographie innerhalb der Wissenschaft sowie auch für die Öffentlichkeit Beifallsbekundungen aus dem Auditorium zur Folge hatte, sorgte der Passauer Oberbürgermeister Jürgen DupPER für Schmunzeln im Saal, als er den „drei Tenören der Passauer Geographie" - gemeint waren die Professoren und Ortsausschuss-Mitglieder Dieter Anhuf, Werner GAMERITH und Ernst STRUCK - jeweils eine Flasche Wein überreichte und meinte, sie müssten keine Sorge haben, dass es sich dabei um einen Passauer Wein handeln könnte, es sei vielmehr ein guter - aus Österreich.

Ergänzt wurde die zentrale Festveranstaltung durch drei weitere Rahmenprogrammpunkte: ein Orgelkonzert im Passauer Dom, eine unter Federführung des Jungen Geographentages organisierte Geo-Party in den großzügig angelegten Räumlichkeiten der Mensa der Universität 
Passau sowie ein Kabarettabend in der Passauer Redoute. Dabei bot der Passauer Kabarettist Rudolf KLAFFENBÖCK sein Programm „GRENZgehen“ dar, indem er eine von ihm selbst in den 1990er Jahren in mehreren Etappen durchgeführte Wanderung und „Spurensuche“ entlang der gesamten österreichischen „Ostgrenze“ (dem Verlauf des ehemaligen Eisernen Vorhangs folgend) auf - auch musikalisch - sehr humorvolle Weise zum Besten gab.

\section{Fazit und Ausblick}

Wenngleich es beim Blick in das Back-Office (vgl. Abb. 2) auch den Anschein hat, dass Michael Heuberger (links im Bild) verzweifelt wäre, kann der von rund 1.500 Personen besuchte 58. Deutsche Geographentag 2013 in Passau aus Sicht des Verfassers vorliegender Ausführungen insgesamt als äußerst gelungen und erfolgreich bezeichnet werden, wozu fachlich interessante und anregende Vorträge und andere Veranstaltungen ebenso beitrugen wie ein abwechslungsreiches Rahmenprogramm und ein generell ansprechender Tagungsort. Das engagierte Passauer Team rund um die Mitglieder des Ortsausschusses und den Kongresskoordinator Michael HeUberger hat sich dafür ein herzliches Dankeschön redlich verdient. In Passau wurde 2013 zweifelsohne der Beweis erbracht, dass es bei guter Kooperation und entsprechendem Zusammenhalt auch kleineren Standorten möglich ist, einen Deutschen Geographentag als Großkongress für Wissenschaft, Schule und Praxis erfolgreich zu veranstalten.

Zwei generelle Aspekte der Entwicklung dieser zentralen Fachtagung der deutschsprachigen Geographie, welche im Zuge des 58. Deutschen Geographentages zu Tage getreten sind, mögen aber durchaus zum Nachdenken anregen: einerseits die Tatsache, dass einige der angekündigt gewesenen Programmpunkte (Vorträge ebenso wie andere Veranstaltungen) schließlich doch nicht stattfinden konnten, sowie andererseits die offensichtliche Unterrepräsentanz der Physischen Geographie speziell im Bereich der Fachsitzungen.

Die Möglichkeit für ein Wiedersehen der deutschsprachigen Geographie-Gemeinschaft wird es Anfang Oktober 2015 in Berlin geben, das in Passau im Rahmen der Fach- und Verlagsausstellung durch einen Stand (vgl. Abb. 5) sowie im Rahmen der zentralen Festveranstaltung vonseiten Elmar KuLKEs als Austragungsort des nächsten und damit insgesamt 59. Deutschen Geographentages angekündigt und präsentiert wurde.

\section{Literaturverzeichnis}

Anhuf D., Gamerith W., Struck E. (2013), Grüß Gott in Passau - der Ortsauschuss [sic!] heißt alle Kongresseilnehmenden [sic!] herzlich willkommen! In: Antwort. Newsletter zum 58. Deutschen Geographentag, 03.10.2013, S. 1. - http://www.geographentag.uni-passau. delfileadmin/documents/Download/ANTWORT_03.10.2013.pdf (22.11.2013).

Musil R. (2009), Der Deutsche Geographentag in Wien 2009 - eine wissenschaftliche Großveranstaltung. In: Mitteilungen der Österreichischen Geographischen Gesellschaft, 151, S. 315-322.

Nissel H., Embleton-Hamann C. (2012), Down to Earth - IGC Cologne 2012. 32. Weltkongress der Geographie in Köln, August 2012. In: Mitteilungen der Österreichischen Geographischen Gesellschaft, 154, S. 341-346.

Rumpolt P.A. (2013), Geographentag 2013 in Passau - ein Rückblick. In: Geographie aktuell. Informationen der Österreichischen Geographischen Gesellschaft, 5, 4, S. 3. 
Mitteilungen der Österreichischen Geographischen Gesellschaft,

155. Jg. (Jahresband), Wien 2013, S. 327-334

\title{
Die Posterausstellung „Alpen - Lebensraum im Wandel “6 DER ÖGG ALS W ANDERAUSSTELLUNG 2011-2013
}

\author{
Peter Alexander RuMPOLT, Wien*
}

mit 6 Abb. im Text

\section{INHALT}

1 Themenschwerpunkt „Lebensraum Alpen“ der ÖGG 2011 als Ausgangspunkt...............327

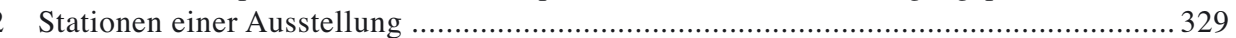

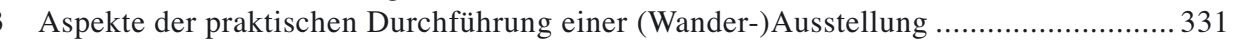

4 Die Kooperationspartner an den einzelnen Standorten .................................................. 331

5 Spezielle Hinweise und Angebote für das Zielpublikum .............................................. 333

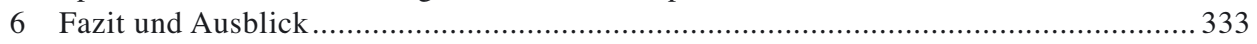

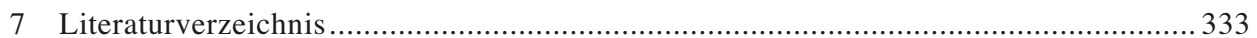

\section{Themenschwerpunkt „Lebensraum Alpen“ der ÖGG 2011 als Ausgangspunkt}

Das 20-jährige Jubiläum der im Jahr 1991 erfolgten Unterzeichnung der Alpenkonvention hat die Österreichische Geographische Gesellschaft (ÖGG), einer Idee ihres Generalsekretärs Robert Musil folgend, zum Anlass genommen, ihre Aktivitäten des Jahres 2011 im Rahmen eines Themenschwerpunkts zum „Lebensraum Alpen“ bestmöglich aufeinander abzustimmen (vgl. auch PEnz 2011, S. 6). Zusätzlich zur entsprechenden fachlichen Schwerpunktsetzung in den „Mitteilungen der Österreichischen Geographischen Gesellschaft“ (MÖGG), Band 153, in den vier Heften des dritten Jahrgangs des ÖGG-Newsletters „Geographie aktuell“ sowie bei Vortragsreihe und Exkursionsangebot der ÖGG (vgl. auch STAUDACHER 2012, S. 436f.) sollte auch eine Ausstellung Teil des Schwerpunktjahres sein.

Ein wesentliches Ziel des thematischen Schwerpunkts bestand darin, Ergebnisse österreichischer geographischer Forschung einer interessierten Öffentlichkeit zu präsentieren (vgl. PeNZ 2011, S. 6) und dadurch einen Diskussionsbeitrag zu leisten (vgl. Musil 2011, S. 1). Dieses Ziel sollte vor allem auch durch die wissenschaftliche Posterausstellung „Alpen - Lebensraum im Wandel" und den begleitend dazu herausgegebenen Sammelband (DitTRICH et al. 2011a) erreicht werden. Die Ausstellung und das Buch zur Ausstellung sind „,als Leistungsschau der Forschungstätigkeiten der österreichischen Geographie im Alpenraum in den letzten Jahrzehnten zu verstehen“ (DitTrich et al. 2011b, S. 6). Dabei wurden einer räumlichen Fokussierung auf

\footnotetext{
* Mag. Peter Alexander Rumpolt, Bundesanstalt Statistik Österreich, Guglgasse 13, A-1110 Wien, und Institut für Geographie und Regionalforschung, Unversität Wien, Universitätsstraße 7, A-1010 Wien; E-Mail: peter.rumpolt@statistik.gv.at, peter.rumpolt@univie.ac.at, http://www.statistik.at,http://geo graphie.univie.ac.at
} 


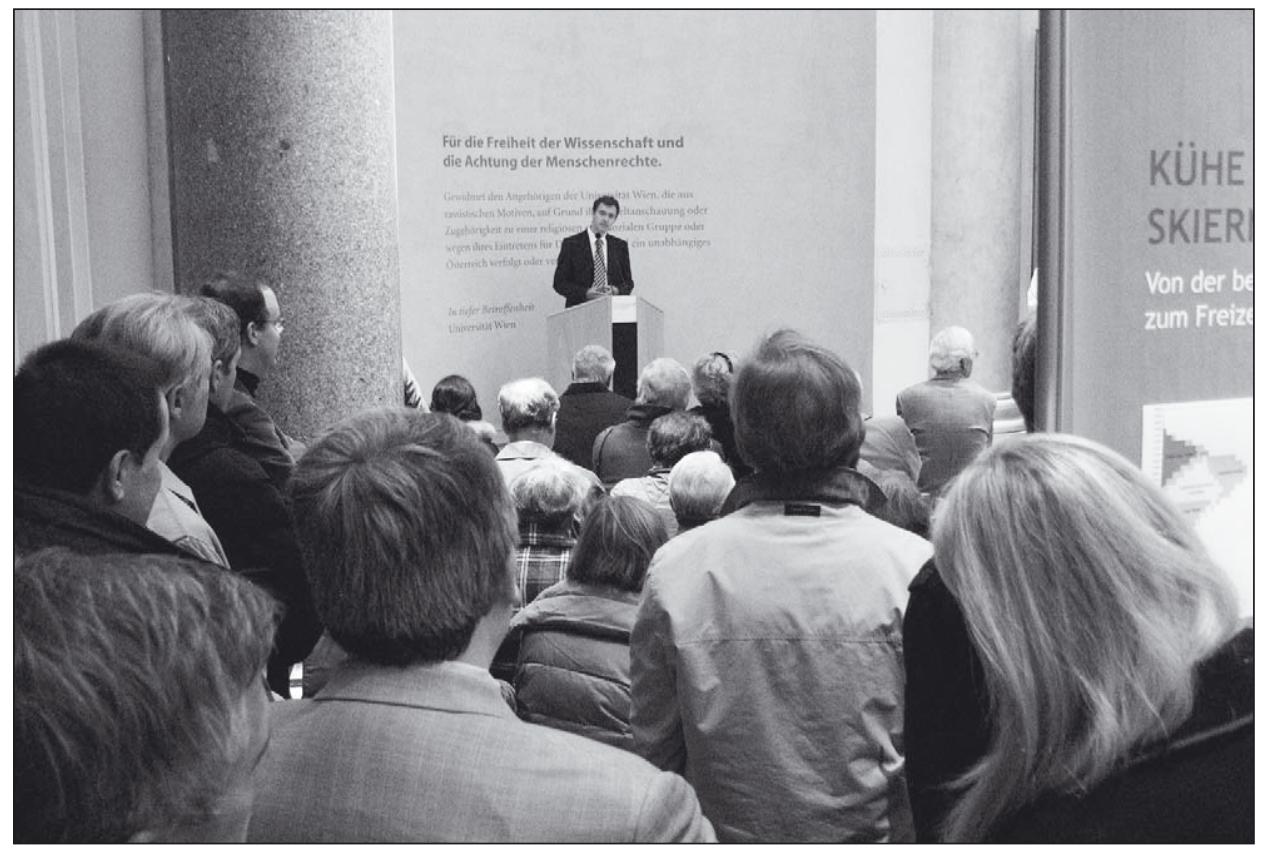

Abb. 1: Robert Musil stellt im Rahmen der Eröffnungsveranstaltung am 25.10.2011 in der Aula der Universität Wien das Ausstellungskonzept vor. (Foto: P.A. RUMPOLT)

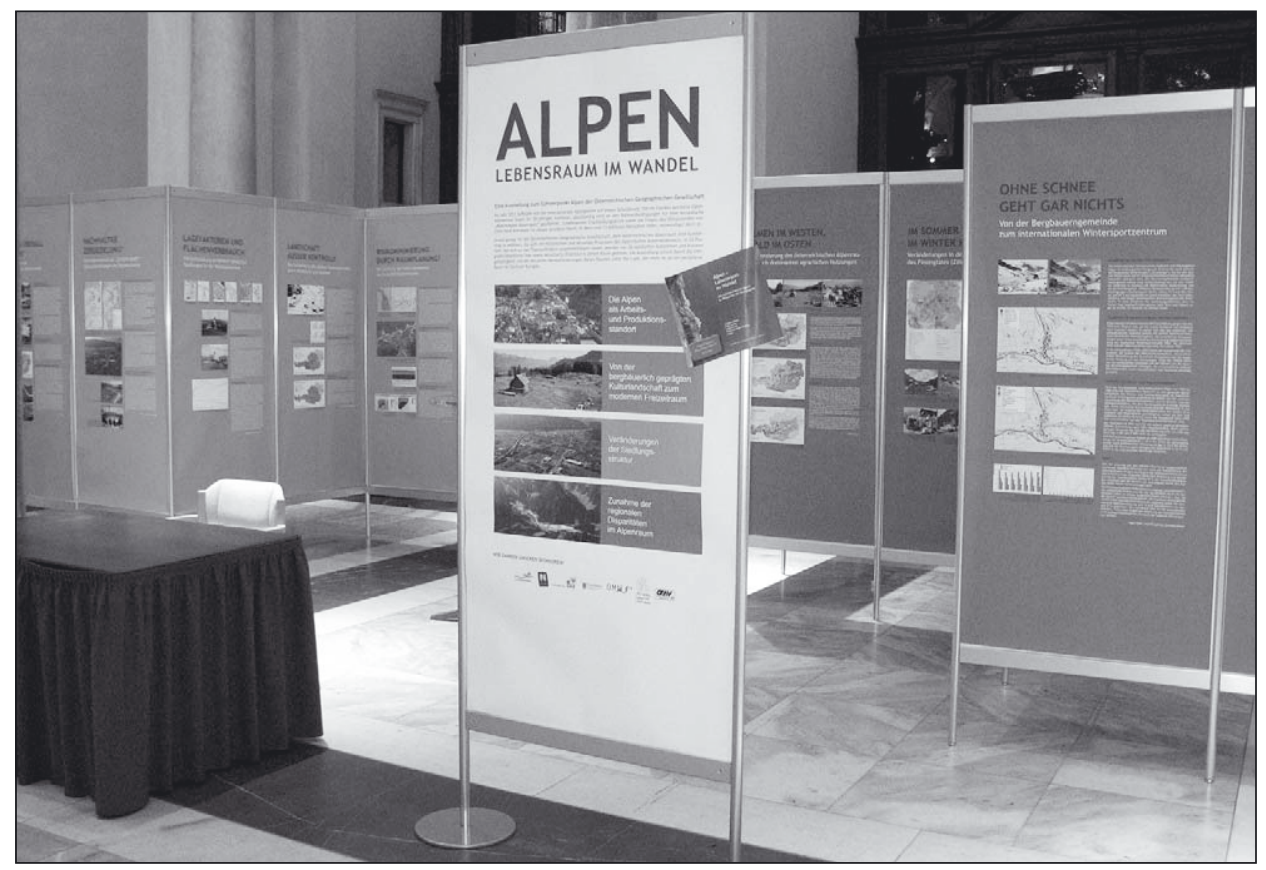

Abb. 2: Erste Präsentation der Ausstellung in der Aula der Universität Wien, Ende Oktober 2011 (Foto: P.A. Rumpolt) 
den österreichischen Alpenraum zahlreiche regionale Fallbeispiele, die Betrachtung historischer und aktueller Prozesse ebenso wie zukünftiger Entwicklungsperspektiven sowie eine große inhaltliche Breite beigestellt (vgl. auch ebd.), welch letztere auch durch die Farbgebung der vier Kapitel der Ausstellung sowie des Sammelbandes zum Ausdruck kommt. Eine Erläuterung des inhaltlichen Konzepts von Buch und Ausstellung findet sich ebenda, eine Buchbesprechung von Werner BätZING ist in den MÖGG, Band 154, erschienen.

Die feierliche Eröffnung der Posterausstellung „Alpen - Lebensraum im Wandel“ erfolgte am 25. Oktober 2011 in der Aula des Hauptgebäudes der Universität Wien (vgl. Abb. 1; vgl. weiters Rumpolt 2011, S. 1); bis 10. November 2011 war die Ausstellung Interessierten dort zugänglich (vgl. Abb. 2). Die Durchführung dieser Ausstellung bedeutete auch das Anknüpfen an eine diesbezüglich lange Tradition von Ausstellungen der ÖGG (vgl. GARTNER \& JORDAN 2006 sowie Musil \& Staudacher 2007).

\section{Stationen einer Ausstellung}

Aus Sicht des Verfassers wäre es schade gewesen, die Ausstellung „nur“ in Wien zu zeigen. Immerhin waren Posterausstellung und Sammelband mit großem Engagement der einzelnen Autorinnen und Autoren, großem (Frei-)Zeiteinsatz und viel „Herzblut“ seitens des „Ausstellungs-Teams“ (Robert Musil, Peter Jordan, Dominik DitTrich, Peter A. Rumpolt) sowie nicht zuletzt auch essenzieller fachlicher und koordinativer Basisunterstützung durch einen der besten Kenner des österreichischen Alpenraumes, Hugo Penz, entstanden. Hugo Penz gebührt dafür ebenso herzlicher Dank wie der im Sommer 2011 als Praktikantin der ÖGG tätigen Agnes Andrea WIENINGER für ihre technische Unterstützung wie für ihre Kreativität in Zusammenhang mit der Formulierung eines Großteils der auf den Postern im Sinne von besonders prägnanten textlichen Aussagen verwendeten „Über-Überschriften“.

Um auch weiteren an der Entwicklung des österreichischen Alpenraumes interessierten Personen den Zugang zu dieser Posterausstellung zu ermöglichen, hat sich der Verfasser bereit erklärt, die ihm vom Vorstand der ÖGG im November 2011 übertragene Aufgabe eines Ausstellungsreferenten der ÖGG für die Dauer von gut zwei Jahren zu übernehmen. Somit konnte die Schlagzeile der letzten Ausgabe von „Geographie aktuell“ des Jahres 2011 folgendermaßen lauten: „Die Alpen-Ausstellung der ÖGG geht auf Tournee!“ (Rumpolt 2011, S. 1). Im Anschluss an entsprechende Vorgespräche und Planungen begann die Geschichte der Posterausstellung „Alpen - Lebensraum im Wandel“" als Wanderausstellung durch Österreich (und darüber hinaus) schließlich am Silvestertag des Jahres 2011 durch den Transport des gesamten Ausstellungssystems von Wien nach Salzburg. Sie hatte in chronologischer Reihenfolge die folgenden Stationen:

25.10.-10.11.2011: Wien, Universität Wien, Hauptgebäude, Aula

09.01 -01 .02.2012: Salzburg, Universität Salzburg, Naturwissenschaftliche Fakultät, Aula

01.09.-21.09.2012: Sankt Gerold, Gemeindeamt, Bewegungsraum [im Rahmen des Kulturfestivals „Walserherbst“]

17.06.-28.06.2013: Bregenz, Vorarlberger Landhaus, Landtagsfoyer

03.10.-06.10.2013: Passau, Universität Passau, Zentralbibliothek, Westflügel [als Sonderveranstaltung 9 des 58. Deutschen Geographentages 2013]

14.10.-17.10.2013: Wien, Universität Wien, Neues Institutsgebäude, 5. Stock, Trakt C (Institut für Geographie und Regionalforschung) 


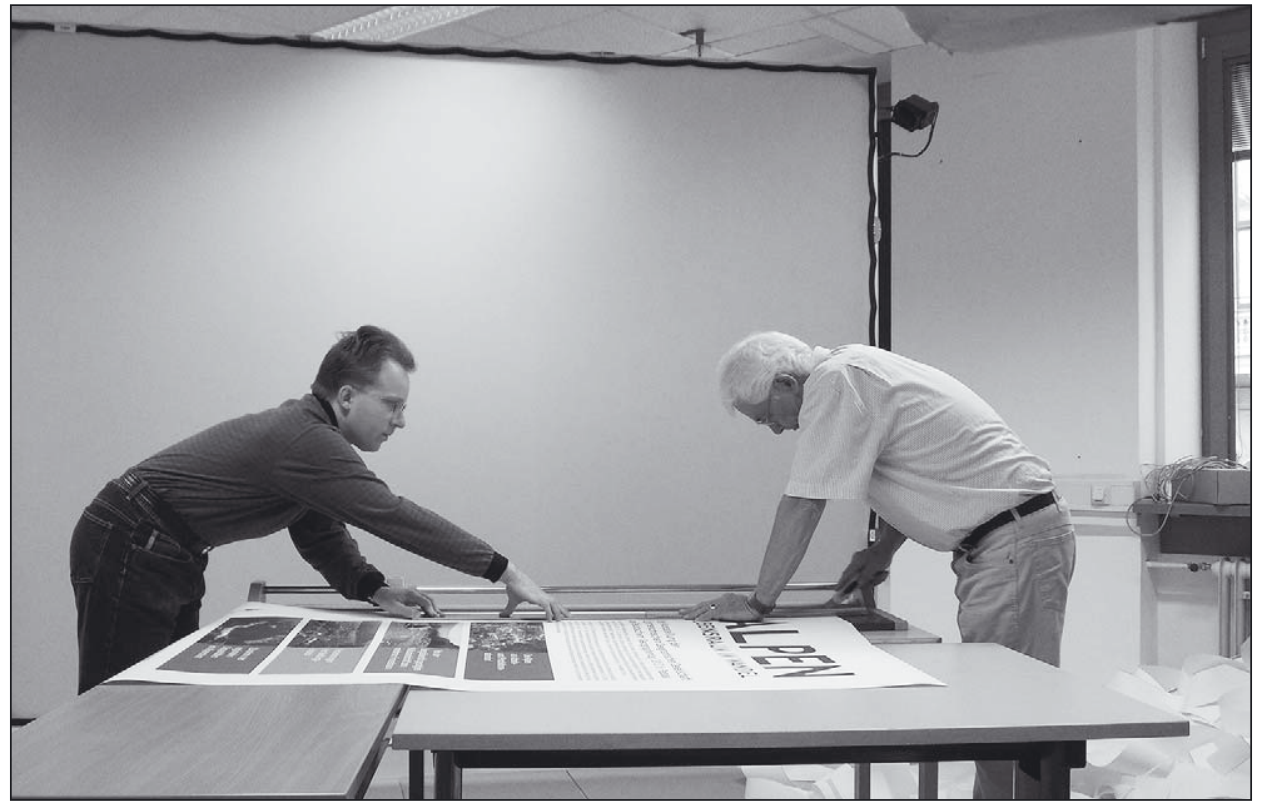

Abb. 3: Peter A. Rumpolt und Christian Staudacher beschneiden Ende September 2013 in Wien die für die Präsentation der Ausstellung in Passau neu ausgedruckten Poster. (Foto: P.A. Rumpolt)

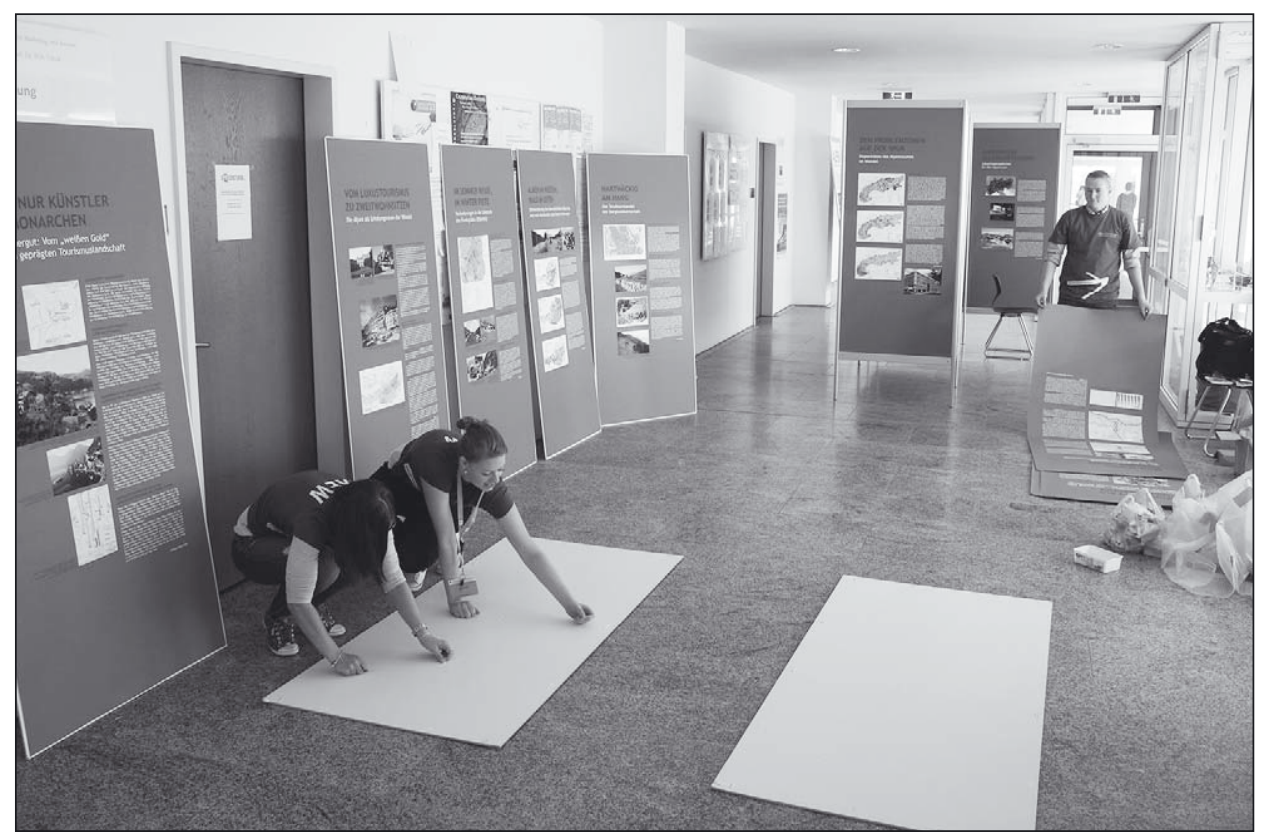

Abb. 4: Natalie Jermann, Julia Zankl und Nils Eger beim Aufbau der Ausstellung in Passau (Foto: P.A. Rumpolt) 


\section{Aspekte der praktischen Durchführung einer (Wander-)Ausstellung}

Um die Präsentation einer solchen Posterausstellung an verschiedenen Standorten zu ermöglichen, sind einige Vorbereitungsschritte erforderlich. So war es beispielsweise notwendig, die einzelnen Poster auf ihren Zustand und ihre potenzielle Wiederverwendbarkeit hin zu untersuchen, bevor der Transport der gesamten Ausstellung an einen weiteren Ausstellungsort in die Wege geleitet wurde. Fallweise wurden einzelne Poster nachgedruckt. Vor dem fünften Standort der Ausstellung (Passau) wurde ein kompletter Neudruck der Poster vorgenommen. Wie schon vor der ersten Präsentation der Ausstellung konnte die Drucklegung der Poster auch im Falle der Nachdrucke jeweils am Institut für Geographie und Regionalforschung der Universität Wien vorgenommen werden. Für die Möglichkeit der Verwendung der Institutsinfrastruktur gebührt Institutsvorstand Thomas GLADE, für die Ausführung Walter LANG herzlicher Dank. Im Anschluss an den Ausdruck war es erforderlich, die Poster auf die für das Wandsystem benötigten Ausmaße exakt zu beschneiden. Dieser Arbeitsschritt, der nicht zuletzt auch aufgrund der Größe der Poster technisches Geschick und Feingefühl erfordert, wurde zumeist vom Präsidenten der ÖGG, Christian StAUDACHER, gemeinsam mit dem Ausstellungsreferenten durchgeführt (vgl. Abb. 3).

In Bezug auf die weiteren Arbeitsschritte gab es zwei unterschiedliche Vorgehensweisen. Die erste Variante fand im Falle des Ausstellungsortes Salzburg Anwendung. Sie bestand darin, dass die Kooperationspartner das Ausstellungssystem selbst (konkret in der Person von Hermann SuIDA) aus Wien abholten und auch den Aufbau der Ausstellung in Salzburg - unter der Federführung von Andreas KocH und Hermann SuIDA - eigenständig vornahmen. Die bei allen weiteren Ausstellungsorten angewandte zweite Variante bestand darin, dass der Ausstellungsreferent der ÖGG den Transport des Ausstellungssystems organisierte, dieser von einer Spedition durchgeführt wurde und der Ausstellungsreferent - mit jeweils tatkräftiger örtlicher Hilfe - die Ausstellung aufbaute. Allen an den Auf- und Abbauten Mitwirkenden gebührt ein spezielles Dankeschön! Im Fall der ersten Variante hatte der örtliche Kooperationspartner (in der Person von Andreas $\mathrm{Koch}$ ) auch um die Ausstellung angefragt, während die Präsentation an allen weiteren Standorten der Wanderausstellung jeweils auf die Initiative des Ausstellungsreferenten der ÖGG zurückging.

\section{Die Kooperationspartner an den einzelnen Standorten}

Voraussetzung für das Gelingen einer Wanderausstellung sind verlässliche Partner an den einzelnen Standorten. In Salzburg war Andreas KocH mit seinem Team der Salzburger Geographischen Gesellschaft (SGG), eines Zweigvereines der ÖGG, und des Fachbereichs Geographie und Geologie der Universität Salzburg ein solcher. Eine ebenso gute Zusammenarbeit war im Falle des dritten Ausstellungsortes (Sankt Gerold im Großen Walsertal) gewährleistet: sowohl mit dem Leiter des Kulturfestivals „Walserherbst“, Dietmar Josef Nigsch, als auch mit dem Bürgermeister von Sankt Gerold, Bruno Summer, und seinem Team (vgl. auch Rumpolt 2012, S. 7). Um die Ausstellung in Bregenz zu ermöglichen, wurden mit der Abteilung Raumplanung und Baurecht (Ansprechpartner Stefan OBKIRCHER) und dem Veranstaltungsmanagement (Ansprechpartner Bernhard KIENER) des Amtes der Vorarlberger Landesregierung entsprechende Vereinbarungen getroffen. Für die Präsentation der Ausstellung im Rahmen des 58. Deutschen Geographentages in Passau wurde mit Werner GAMERITH und Michael Heuberger vom Fachbereich Geographie der Universität Passau eine Kooperation eingegangen. Ansprechpartner für den Aufbau der Ausstellung am Institut für Geographie und Regionalforschung der Universität Wien waren Thomas GLADE und Rudolf VoIT. 


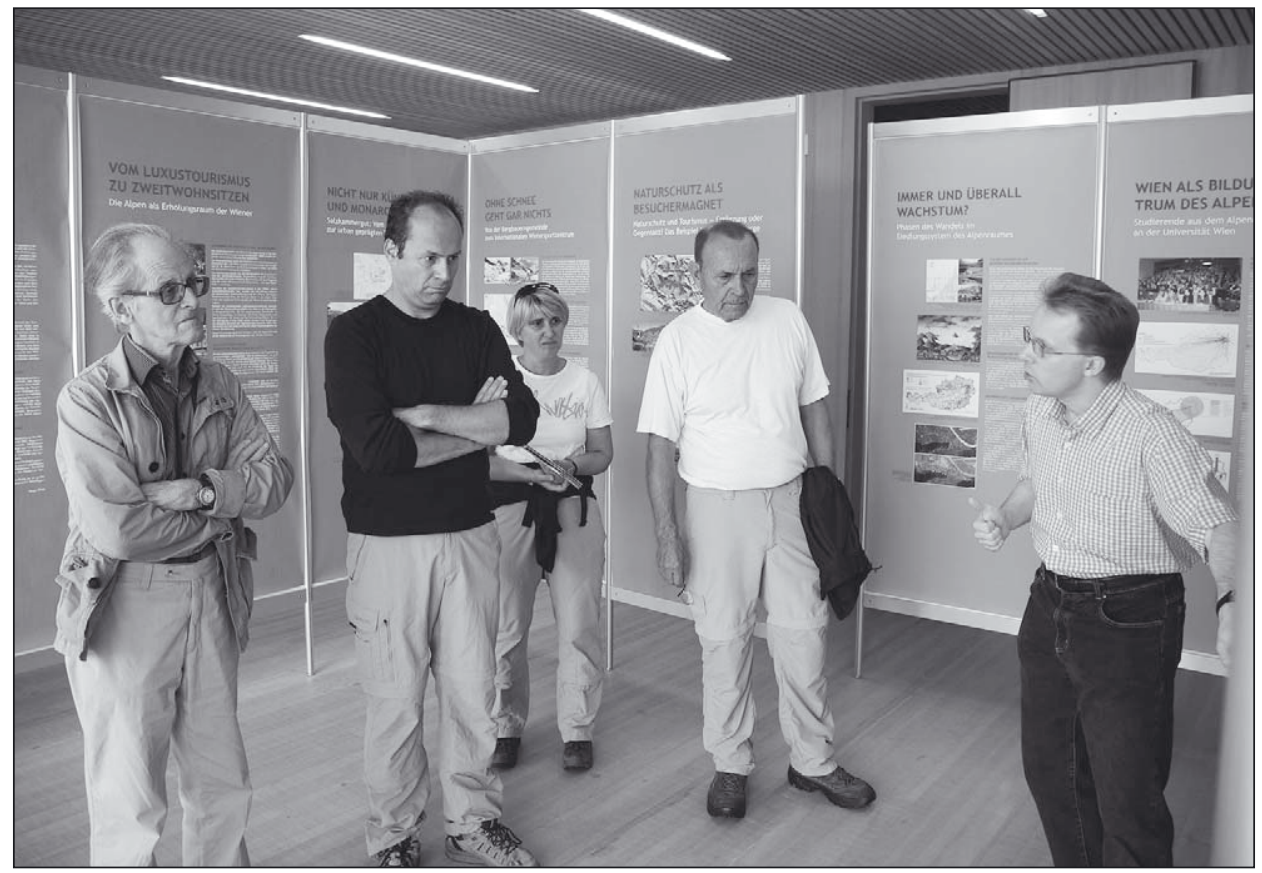

Abb. 5: Peter A. Rumpolt führt durch die Ausstellung in Sankt Gerold. (Foto: V. Plieninger)

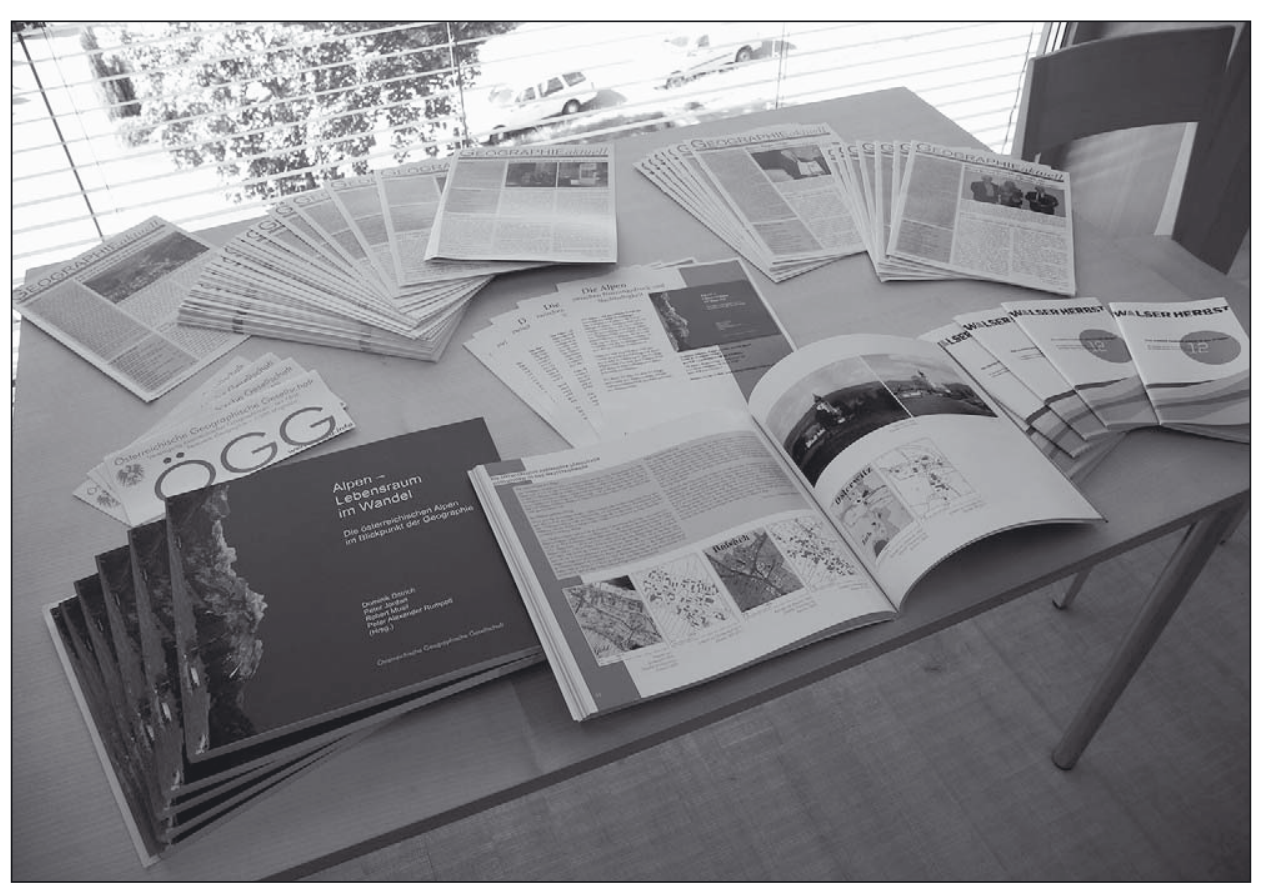

Abb. 6: Der Sammelband zur Ausstellung sowie weitere Informationsmaterialien in Sankt Gerold (Foto: P.A. RuMPOLT) 


\section{Spezielle Hinweise und Angebote für das Zielpublikum}

Um die interessierte Öffentlichkeit zu erreichen, wurden zusätzlich zu entsprechenden Hinweisen im ÖGG-Newsletter „Geographie aktuell“, auf der Homepage der ÖGG und auf den Webseiten beteiligter Universitätsinstitute oder -fachbereiche auch weitere Informationskanäle bedient. So wurde beispielsweise über die Ausstellung in Salzburg auch im Regionalradio berichtet. Die Ausstellung in Sankt Gerold bildete einen eigenen Programmpunkt im Rahmen des Kulturfestivals „Walserherbst“ des Jahres 2012, welcher auch in dem in Vorarlberg weit verbreiteten Programmheft des Festivals als solcher ersichtlich war. Anlässlich der Ausstellung in Bregenz wurde seitens des Amtes der Vorarlberger Landesregierung am 24.06.2013 eine eigene Presseaussendung herausgegeben. Im Rahmen des 58. Deutschen Geographentages 2013 in Passau (vgl. diesbezüglich auch Rumpolt in diesem Band sowie Rumpolt 2013, S. 3) bildete die Alpen-Ausstellung der ÖGG eine eigene Sonderveranstaltung, welche als solche sowohl auf der Webseite als auch im Programmheft und im Newsletter des Geographentages angekündigt wurde. Ein weiteres Mittel der Ankündigung der einzelnen Standorte der Wanderausstellung bildeten zielgruppenspezifische E-Mail-Aussendungen.

Zusätzliche Angebote gab es 2011 in Wien sowie speziell auch 2012 in Salzburg und Sankt Gerold. Während die Ausstellung in Wien am 25.10.2011 im Rahmen einer eigenen Eröffnungsveranstaltung mit Worten von Robert MusiL, Hugo Penz und Christian STAUdACHER eingeleitet wurde (vgl. Abb. 1), hat Andreas Koch zu Beginn des Jahres 2012 in Salzburg eigene Führungen für Schulklassen ermöglicht. Am Eröffnungswochenende in Sankt Gerold (01.-02.09.2012) wurden geführte Besichtigungen der Ausstellung angeboten, durchgeführt vom Autor dieses Artikels (vgl. Abb. 5). An allen Standorten lag auch der Sammelband zur Ausstellung auf (vgl. Abb. 6).

\section{Fazit und Ausblick}

Mit dem Themenschwerpunkt „Lebensraum Alpen“ des Jahres 2011 im Allgemeinen sowie mit der Posterausstellung und dem dazugehörigen Sammelband „Alpen - Lebensraum im Wandel“ im Speziellen wurde seitens der Österreichischen Geographischen Gesellschaft der Versuch unternommen, Ergebnisse geographischen Forschens einer möglichst breiten Zielgruppe auf möglichst anschauliche Weise wissenschaftlich näher zu bringen. Die Präsentation der Ausstellung an verschiedenen Standorten hat diesen Effekt sicherlich verstärkt.

Zahlreiche positive Rückmeldungen zu Ausstellung und Sammelband lassen den Schluss zu, dass diese Form der Weitergabe von Wissen gerade für wissenschaftliche Gesellschaften zukunftsweisend ist.

\section{Literaturverzeichnis}

Dittrich D., Jordan P., Musil R., Rumpolt P.A. (Hrsg.) (2011a), Alpen - Lebensraum im Wandel. Die österreichischen Alpen im Blickpunkt der Geographie. Wien, Österreichische Geographische Gesellschaft.

Dittrich D., Jordan P., Musil R., Rumpolt P.A . (2011b), Vorwort der Herausgeber. In: DitTrich D., Jordan P., Musil R., Rumpolt P.A. (Hrsg.), Alpen - Lebensraum im Wandel. Die österreichischen Alpen im Blickpunkt der Geographie, S. 6-7. Wien, Österreichische Geographische Gesellschaft.

Gartner G., Jordan P. (2006), Ausstellungen der Österreichischen Geographischen Gesellschaft (ab 1959) und ihrer Vorgängergesellschaften (1856-1959). In: KRETSCHMER I., FASCHING 
G. (Hrsg.), Österreich in der Welt, die Welt in Österreich. Chronik der Österreichischen Geographischen Gesellschaft. 150 Jahre (1856-2006), S. 313-334. Wien, Österreichische Geographische Gesellschaft.

Musil R. (2011), Alpenraum im Fokus: Zwischen Nachhaltigkeit und Erschließungsansprüchen. In: Geographie aktuell. Informationen der Österreichischen Geographischen Gesellschaft, 3,3,S.1.-http://oegg.oeaw.ac.at/publikationen/gg_aktuell/geo_aktuell_9_2011_online. $p d f(22.11 .2013)$.

Musil R., Staudacher C. (2007), Mensch. Raum. Umwelt. Einblicke in die Geographie - Ausblicke in die Welt. Posterausstellung in der Säulenhalle des Parlaments, 5.-19. Oktober 2006. In: Kretschmer I. (Hrsg.), Das Jubiläum der Österreichischen Geographischen Gesellschaft. 150 Jahre (1856-2006), S. 181-214. Wien, Österreichische Geographische Gesellschaft.

Penz H. (2011), Lebensraum Alpen. In: Geographie aktuell. Informationen der Österreichischen Geographischen Gesellschaft, 3, 1, S. 6. - http://oegg.oeaw.ac.at/publikationen/gg aktuell/geo_aktuell_1_2011_online.pdf (22.11.2013).

Rumpolt P.A. (2011), Die Alpen-Ausstellung der ÖGG geht auf Tournee! In: Geographie aktuell. Informationen der Österreichischen Geographischen Gesellschaft, 3, 4, S. 1. - http:// oegg.oeaw.ac.at/publikationen/gg_aktuell/geo_aktuell_10_2011_online.pdf(22.11.2013).

Rumpolt P.A. (2012), Posterausstellung zu Gast in Vorarlberg. In: Geographie aktuell. Informationen der Österreichischen Geographischen Gesellschaft, 4, 3, S. 7. - http://oegg. oeaw.ac.at/publikationen/gg_aktuell/geo_aktuell_3_2012_online.pdf (22.11.2013).

Rumpolt P.A. (2013), Geographentag 2013 in Passau - ein Rückblick. In: Geographie aktuell. Informationen der Österreichischen Geographischen Gesellschaft, 5, 4, S. 3.

Staudacher C. (2012), Gesellschaftsnachrichten. In: Mitteilungen der Österreichischen Geographischen Gesellschaft, 154, S. 433-447. 